DOI: 10.1016/j.ijpe.2015.10.008

\title{
Production control problem integrating overhaul and subcontracting strategies for a quality deteriorating manufacturing system
}

\author{
HÉctor Rivera-Gómez ${ }^{1}$, Ali GHARbi ${ }^{2}$, JEAN-PIERRE KENNÉ ${ }^{3}$, \\ Oscar Montaño-Arango ${ }^{1}$, Eva SELENE HERNANDEZ-Gress ${ }^{1}$ \\ ${ }^{1}$ Academic Area of Engineering, Autonomous University of Hidalgo, \\ Pachuca-Tulancingo Road km. 4.5, City of Knowledge, Mineral de la Reforma, Hgo, \\ Mexico, 42184, hriver06@hotmail.com, oscarma11@hotmail.com, \\ evaselenehg@hotmail.com \\ ${ }^{2}$ Automated Production Engineering Department, École de technologie supérieure, \\ Production System Design and Control Laboratory, Université du Québec \\ 1100 Notre Dame Street West, Montreal, QC, Canada, H3C 1K3 \\ ali.gharbi@etsmtl.ca \\ ${ }^{3}$ Mechanical Engineering Department, École de technologie supérieure, \\ Laboratory of Integrated Production Technologies, Université du Québec \\ 1100 Notre Dame Street West, Montreal, QC, Canada, H3C 1K3 \\ jean-pierre.kenne@etsmtl.ca
}

\begin{abstract}
We study an unreliable deteriorating manufacturing system that produces conforming and nonconforming parts to satisfy a constant demand product rate. The manufacturing system is comprised of a failure-prone machine. Due to the combined effect of random availability variations and deterioration, the system is not able to fulfill long-term product demand. In particular, when finished goods inventory is positive, clients demand are fulfilled on-time and without delay. When backlog exists, subcontracting options are available at a higher cost to supplement the limited production capacity of the manufacturing system. The effect of deterioration is observed mainly in the quality of the parts produced, since the rate of defectives increases as the machines deteriorate. Overhaul activities can be conducted to mitigate the effect of deterioration. We propose a joint feedback control policy based on a stochastic dynamic programming formulation which aims simultaneously to determine the production and overhaul rates, and the rate at which subcontractors are requested. The proposed joint control policy minimizes the total cost, including the inventory, backlog, repair, overhaul, defectives, production and subcontracting costs, over an infinite planning horizon. To determine the optimal control parameters, we adopt a numerical scheme to solve the optimality equations and a numerical example is presented as an illustration of the approach. The structure of the joint control policy is validated through an extensive sensitivity analysis.
\end{abstract}

Keywords: Quality, Manufacturing System, Production, Subcontracting, Deteriorating systems. 


\section{Introduction}

In modern organizations, quality and production planning are critical for market survival. Typically, a number of disruptions such as deterioration, delays, defectives, failures, etc., limit the organization's ability to satisfy product demand without delays, leading to increased lead times and customer dissatisfaction. Therefore, manufacturing systems must be designed efficiently to respond quickly to such disruptions, mainly among manufacturers with limited capacity. One attractive option is to increase capacity to reduce the lead time through the use of subcontractors. The cost associated with subcontractors can be justified by the ensuing reduced lost sales and required inventory. Furthermore, maintenance strategies are available to improve the system performance. Unfortunately, more research is needed to have a better understanding of the connection between production and quality, as well as to include the effect of maintenance and subcontracting issues in analyzing such relationships. In this study, we therefore aim to propose an integrated model that defines the structure of an optimal joint policy for the interaction of production, quality, maintenance and subcontracting activities in the context of deterioration.

Closed-form solutions were available for the production control problem with the work of Akella and Kumar [1], who addressed the case of a single-machine, one-part type considering a discounted cost; as well they determined the structure of the so-called Hedging Point Policy. Since quality is a major factor in competitiveness in current markets, recent extensions of this research area include connections to quality issues. For instance, Inman et al. [2] highlighted the importance of the interaction between production and quality issues, and reviewed the limited literature on the intersection of quality and production system design. Colledani et al. [3] revised problems, methods and tools to support the strong interaction among production logistics, quality and maintenance functions; also they highlighted the main challenges and opportunities for zero-defect oriented manufacturing industries. Mathematical models integrating production and quality issues were presented in Kim and Gershwin [4, 5] where they determined several performance metrics for small and larger systems. Other analytical models were introduced in the work of Colledani et al. [6], who proposed an integrated quality and production logistics model, which significantly reduced the output fraction of defectives. With this model improved performance is achieved towards existing solutions, which deal only with quality aspects. Further, some authors have incorporated quality issues in the production policy. For instance, Radhoui et al. [7] developed a model for lots that are subject to quality control and determined the maintenance strategy and the optimal buffer size. Analytical expressions for the production control policy and the related total cost are presented in Mhada et al. [8], for a failure-prone machine whose final product includes some defective parts. Defective production is also covered in the work of Bouslah et al. [9] in which an acceptance sampling plan is used to control the quality of the lots produced. They jointly optimize the batch size, the hedging level and the sample size. Additionally, Dhouib et al. [10] presented an integrated strategy for the production inventory control and preventive maintenance for a manufacturing system with imperfect production, using an age-based preventive maintenance policy to reduce the shift rate to the out-of-control state. We should note that the above works have a restrictive assumption, regarding the rate of defectives which is assumed to be constant 
for the entire time horizon. Obviously, this might not be true in practice, since it is very common for manufacturing systems to experience the accumulated effect of deterioration, which has serious impacts on several parameters of the system. The deteriorating systems field may therefore provide an appealing approach for our research.

As a matter of interest deterioration has been a major topic in the manufacturing systems field. For example, Martinelli and Piedimonte [11] described an optimal backlog/inventory problem for a production system whose deterioration depends on its production rate. A combination of the replacement planning problem with production control and preventive maintenance was presented in Dehayem et al. [12], where they focused on a manufacturing system that experienced a double deterioration caused by the age of the machine and by imperfect maintenance. A key observation to be made here is that the aforementioned papers analyze the effect of deterioration on increasing failure rates or decreasing production rates, while disregarding its effect on quality. The influence of deterioration on part quality was covered for the case of degrading machines in Colledani and Tolio [13], who tackled the production rate of conforming parts of deteriorating machines controlled by preventive maintenance and control charts. Additionally, a more detailed discussion about quality deterioration can be found in Rivera-Gómez et al. [14], who determined production and major maintenance strategies for a manufacturing system with increasing defectives caused by the influence of deterioration. In spite of these primary attempts to relate deterioration with part quality, we believe that more research is required in order to fully understand its effect on the control policy, mainly because, in real production, manufacturers encounter a wide variety of phenomena. Additionally, it is important to use effective measures to palliate limited capacity, and to that end, one option is the use of subcontracting to ensure ontime fulfillment of product demand.

Utilizing subcontractors can be a possible option for production systems with limited capacity. Tan and Gershwin [15] derived a feedback control policy that determines the production rate and the rate at which subcontractors are required to deliver products for a random demand scenario. Another subcontracting model was presented in Hajej et al. [16], who obtained an optimal production plan and preventive maintenance program, where products returned by customers are sent to a subcontractor for recycling and remanufacturing. An adjustable capacity for an unreliable manufacturing system was treated in the work of Gharbi et al. [17], in which a reserve machine is called upon as support to satisfy the product demand if the inventory level falls below a specific threshold. Recently, Dror et al. [18], proposed a methodology to determine safety-stock level and storage capacity for a multiple manufacturing facilities multiple product with subcontracting options. An extension of this model was done by Assid et al. [19] who tested different subcontracting policies. One important remark that can be made here concerns the fact that none of the works mentioned above considered subcontracting actions in the context of progressive limited capacity. Indeed, in these works, subcontracting is not studied in the context of machine wear causing a continuous deterioration of the part quality.

Following the above discussion, we can observe that the purpose of this study is to develop a stochastic optimal control model allowing us to extend previous contributions, 
such as $[14,17,18]$, in three important directions. First, we cover the impact of quality issues on the production control policy, in a bid to further analyze the relationship between production and quality. Second, we analyze the impact of the progressive wear of the manufacturing system, which leads to a Semi-Markov model where the property of memoryless holds for the transition failure rate. However, we keep memory of the age of the machine to model quality deterioration. Third, with subcontractors, we provide the manufacturer with external product sources. When the demand exceeds the capacity of the manufacturing system, subcontractors may be useful for reducing backlog, improving customer service, and reducing the number of lost sales. Also, overhaul activities can be conducted to mitigate the effect of deterioration. One of our goals is to minimize the total incurred cost over an infinite planning horizon comprising the inventory, backlog, repair, overhaul, defectives, production and subcontracting costs. To the best of our knowledge, this set of characteristics has not been covered jointly in the literature. We use HamiltonJacobi-Bellman equations to determine the structure of the control policy and the results obtained are further examined in a sensitivity analysis.

The rest of the paper is organized as follows. The industrial context of the paper is presented in Section 2. The model description and its assumptions are given in Section 3. The control problem formulation is presented in Section 4. The joint control policy is detailed in Section 5. Additionally, the evaluation of the control policy is described in Section 6 through a sensitivity analysis comprising the effect of cost and system parameters variation. Section 7 discusses about some managerial implications for the obtained results. Section 8 concludes the paper.

\section{Industrial context}

The model presented in this paper is suitable for many industries characterized by deterioration in which machines are unreliable and their production rates can be controlled. Examples of such systems include machining tools (i.e. machining centers, grinders, milling, etc) typically comprised by a large number of components which stochastically deteriorate over time, as remarked in Dehayem et al. [20]. The phenomenon of deterioration is common in the automobile, aircraft, machine tools and paper manufacturing plants (Kouedeu et al. [21]). The typical approach with deterioration in many industrial and academic studies has been to focus on its effect on the system's reliability, as in Rosenblatt and Lee [22], Sana [23] and Colledani and Tolio [24].

In a realistic context, deterioration also limits the production capacity of a manufacturing system; hence many firms must react quickly to changes in the demand by carrying some inventory or increase its capacity by subcontracting. Thus, this paper has many applications in several sectors, such as the textile, retail channel, pharmaceutic, semiconductor, etc. Normally, in situations where the production system has a limited capacity, subcontracting is used to achieve capacity flexibility to gain and maintain a competitive advantage. More specifically, our paper is partly in line with the work of Dror et al. [18], in the sense to study a real production system with limited production capacity which uses subcontracting to avoid backlog, reduce inventory and improve lead time. However, Dror et al. [18] disregarded the effect of deterioration; unfortunately their 
system is subject to a deterioration process with severe effects of the performance of the machine. This deterioration could also affect products' quality. In the next section, we formulate a novel stochastic optimal control model and apply appropriate techniques for its solution.

\section{Notation and problem description}

In this section, we present the notation and the problem statement of the control problem under study.

\subsection{Notation}

The following notations are required for the proper formulation of the proposed model:

$\begin{array}{ll}x(t) & \text { Inventory level at time } t \\ a(t) & \text { Age of the machine at time } t \\ d & \text { Constant demand rate of products } \\ \xi(t) & \text { Stochastic process } \\ Q(\cdot) & \text { Transition rate matrix of the stochastic process } \\ u_{1}(\cdot) & \text { Production rate of the manufacturing system } \\ u_{2}(\cdot) & \text { Subcontracting rate } \\ \mathrm{u}_{1}^{\max } & \text { Maximum production rate of the machine } \\ \mathrm{u}_{2}^{\text {max }} & \text { Maximum production rate for subcontracting } \\ \beta(\cdot) & \text { Rate of defectives } \\ \rho & \text { Discount rate } \\ \pi_{i} & \text { Limiting probability at mode } i \\ q_{\alpha \alpha^{\prime}}(\cdot) & \text { Transition rate from mode } \alpha \text { to mode } \alpha \\ \gamma^{(t)}(\cdot) & \text { Cost rate function associated with the process } \xi(t) \\ J(\cdot) & \text { Expected discounted cost function } \\ v(\cdot) & \text { Value function } \\ \omega_{o}(\cdot) & \text { Control variable for the overhaul activity } \\ \omega_{\min } & \text { Minimum overhaul rate } \\ \omega_{\max } & \text { Maximum overhaul rate } \\ \tau & \text { Jump time of } \xi(t) \\ c^{+} & \text {Inventory holding cost / units / time unit } \\ c^{-} & \text {Backlog cost / units / time unit } \\ c_{r} & \text { Repair cost } \\ c_{o} & \text { Overhaul cost } \\ c_{d} & \text { Cost of defectives } \\ C_{M 1} & \text { Cost of production of the manufacturing system per unit of } \\ C_{M 2} & \text { produced parts } \\ \theta & \text { Cost of production of subcontracting per unit of produced parts } \\ & \text { Adjustment parameter for the rate of defectives } \\ & \end{array}$




\subsection{Problem description}

Without loss of generality, we consider the case a manufacturing system producing one part type to gain more insight about the effect of deterioration in the system. Additionally, the manufacturing system $\left(\mathrm{M}_{1}\right)$ presented in block diagram of Figure 1, is unreliable because it is subject to random failures. Initially, the manufacturing system can satisfy the demand, but because of the severe effects of its progressive deterioration, the machine eventually begins to wear, decreasing the quality of the parts produced and increasing the rate of defectives. Since product demand is satisfied only with flawless parts, when the manufacturing system reaches a certain level of deterioration, it is no longer capable of fulfilling product demand by its own. In this context of progressive deterioration, subcontracting $\left(\mathrm{M}_{2}\right)$ is an available option to satisfy the part of the demand that the manufacturing system is not capable of fulfilling, as illustrated in Figure 1. However, the use of subcontracting is limited because it involves a higher cost than the manufacturing system $\left(\mathrm{M}_{1}\right)$. Additionally, major maintenance activities, also known as overhaul, can be conducted on the manufacturing system to reduce the effects of deterioration and restore the rate of defectives to initial conditions. To complement the description of the system, we seek in our integrated model to determine the optimal control policy that includes the optimal production plan for the manufacturing system, the optimal policy for the subcontracting option, as well as the optimal overhaul strategy minimizing the total incurred cost. In this context, the total cost is composed of several categories such as the inventory, backlog, repair, overhaul, defectives, production and subcontracting costs.

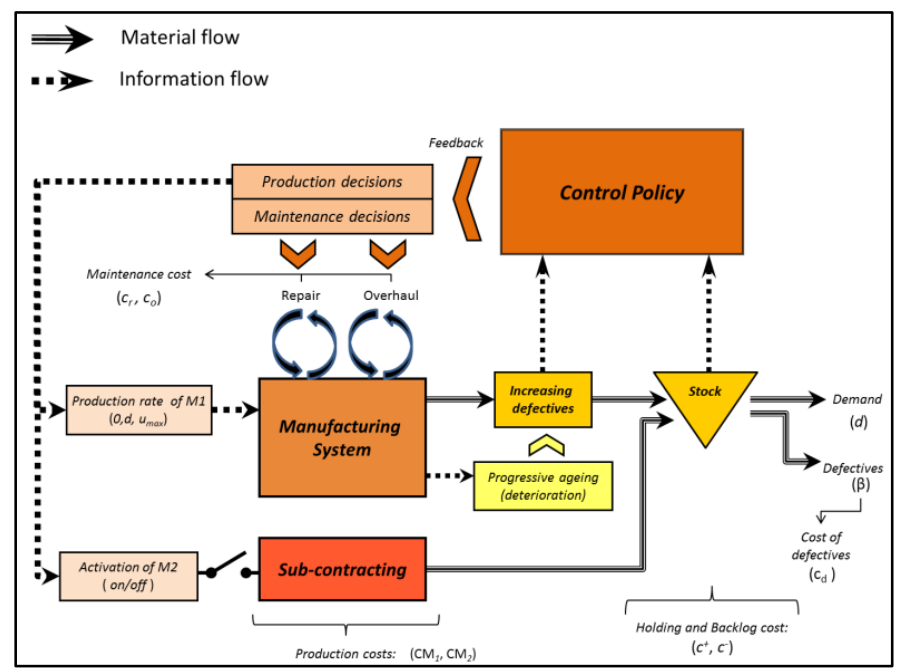

Figure 1: Block diagram of the proposed production system

\section{Model formulation}

We begin this section by formulating a stochastic model based on the series of features discussed in the previous section. The dynamics of the manufacturing system is described 
by a three-mode stochastic process $\xi(t) \in \Omega=\{1,2,3\}$. When $\xi(t)=1$, the manufacturing system is operational, producing a mix of flawless and defectives products. When $\xi(t)=2$, the manufacturing system is in failure mode, where a minimal repair is conducted, leaving the manufacturing system in as bad-as-old (ABAO) conditions before failure. Lastly, when $\xi(t)=3$, the manufacturing system is in the overhaul mode, where a perfect maintenance is carried out, restoring the machine to asgood-as-new (AGAN) conditions, and palliating completely the effects of deterioration.

The manufacturing system evolves according to a continuous-time discrete-state SemiMarkov process, given that it remains in ABAO conditions after repair activities and returns to AGAN conditions after overall activities. An ABAO corrective maintenance leads to a memory process depending on the repair activities while the perfect maintenance (AGAN), provide a memoryless property to dynamics of the system. We then describe the dynamics of the overall system by a semi-markovian process which is a sequential combination of $\mathrm{ABAO}$ and AGAN actions. The manufacturing system may stay in any of the three modes over an infinite horizon. The Semi-Markov process implies a generator matrix $Q$ such that $Q=\left\{q_{\alpha \alpha^{\prime}}\right\}$, where $q_{\alpha \alpha^{\prime}}$ indicates the transition rate from mode $\alpha$ to $\alpha^{\prime}$, and $q_{\alpha \alpha^{\prime}} \geq 0, q_{\alpha \alpha^{\prime}}=-\sum_{\alpha^{\prime} \neq \alpha} q_{\alpha \alpha^{\prime}}$ and $\alpha, \alpha^{\prime} \in \Omega$.

A number of researchers assert that the quality of the parts produced is affected by the deterioration of the manufacturing systems, as suggested by Kim and Gershwin [4] and addressed by Colledani an Tolio [24], thus defining the relationship between deterioration and quality. Furthermore, in the deteriorating systems domain, the age of the machine is commonly employed to define the level of deterioration of the system, as in Love et al. [25], thus implying an age-deterioration relationship. Hence, based on the relationships between deterioration-quality and age-deterioration, the rate of defectives can directly be related with the age of the machine. These observations lead us to define an age-quality relationship, which in this paper, is modeled with the following increasing function:

$$
\beta(a)=b_{1}+b_{2}\left(1-e^{-k_{2} \cdot \theta \cdot\left[a(t)^{3}\right]}\right)
$$

where $\theta$ is an adjustment parameter for the speed of deterioration of the rate of defectives, as exemplified in Figure 2 ( $\theta$ varies in the interval $0 \leq \theta \leq 1), b_{1}$ is the initial value of the rate of defectives, $b_{2}$ represents the upper limit and $k_{2}$ indicates a given constant. Note that when $\theta \rightarrow 1$, the manufacturing system deteriorates faster, producing more defectives, and when $\theta \rightarrow 0$, the machine slows down its deterioration rate, producing defectives at a lower pace. Maintenance service and quality data are the source for determining the value of the parameters $b_{1}, b_{2}, k_{2}, \theta$ for a particular system. Equation (1) is an increasing function used in this paper to model the impact of the deterioration on the dynamics of the machine. For manufacturing systems such as machine tools (i.e. milling machine, lathe, etc.), we can design appropriate maintenance policies, with expression similar to Equation (1), as noted in Kenne and Gharbi [26], Njike et al. [27] and BenSalem et al [28]. 


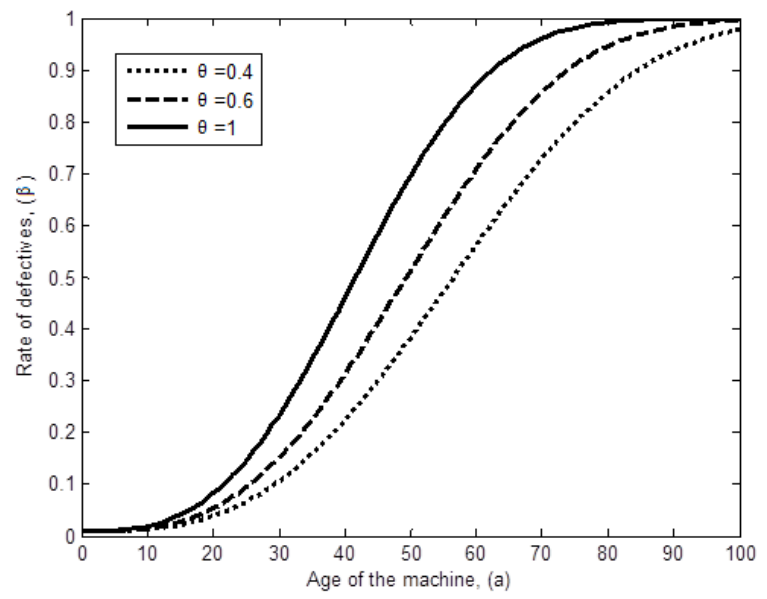

Figure 2: Variation of the rate of defectives for different values of the parameter $\theta$

It should be stated that the manufacturing system studied is subject to deterioration, and its effect is mainly reflected on the quality of the parts produced, increasing the rate of defectives $\beta$. Therefore, we consider that the dynamic behavior of the stock level evolves according to the following differential equations:

$$
\dot{x}(t)=u_{1}(t, \alpha) \cdot[1-\beta(\mathrm{a})]+u_{2}(t)-d, \quad x(0)=x_{0},
$$

In this expression, $x_{0}$ refers to the initial inventory level, $a$ is the age of the manufacturing system, and $\beta(a)$ represents the rate of defectives as a function of the age. In Equation (2), we notice that only the manufacturing system produces defectives, while the subcontracting option, $u_{2}(t)$, is free of this disruption since it is assumed that subcontractors provide flawless parts to complete product demand. To complement the formulation, we define the age of the manufacturing system at time $t$ as a function of its production rate since the last restart, with the following differential equation:

$$
\dot{a}(t)=k_{1}\left[u_{1}(t)\right], \quad \mathrm{a}(\mathrm{T})=0
$$

where $k_{1}$ denotes a positive constant, and $T$ is the last restart time of the machine following overhaul activities. Further, $u_{1}(\cdot)$ is the production rate of the machine and $\mathrm{u}_{1}^{\max }$ is the maximum production of the machine, with:0 $\leq u_{1}(\cdot) \leq \mathrm{u}_{1}^{\max }$.

We assume in the model, that initially the machine has sufficient capacity to satisfy customer's demand but cannot satisfy the demand by its own when the machine is highly deteriorated. Therefore, subcontracting can be used as a short-term option to increase capacity and satisfy the part of the demand that is not satisfied by the manufacturing system. Thus, at time $t$, the machine request subcontracting to supply material at rate $u_{2}(\cdot) \in\left[0, \mathrm{u}_{2}^{\max }\right]$, as follows: 


$$
u_{2}(\cdot)=\left\{\begin{array}{c}
\mathrm{u}_{2}^{\max } \\
d \\
d-u_{1}(\cdot) \cdot[1-\beta(a)] \\
0
\end{array}\right.
$$

where $\mathrm{u}_{2}^{\max } \geq \mathrm{u}_{1}^{\max }>d$, the above equation means that the subcontracting rate may be any of the following four options: operational at the maximum subcontracting rate, producing at the demand rate, producing the difference of demand that the manufacturing system is not able to satisfy, and not producing. At time $t$, the machine has an initial capacity, $u_{1}^{\max }$, let $\pi_{1}$ denote the steady state availability of the manufacturing system, defined by $\pi_{1}=1 /\left(1+q_{12} / q_{21}\right)$. Further, given the effect of deterioration on the machine, we rely more and more on subcontracting to fulfill the product demand. Thus, the whole capacity constraint of the system, taking into account deterioration and subcontracting, is:

$$
\mathrm{u}_{1}^{\max }(\cdot) \cdot[1-\beta(a)] \cdot \boldsymbol{\pi}_{1}+\mathrm{u}_{2}^{\max }(\cdot) \geq d
$$

Note that if the condition $u_{1}^{\max }(\cdot) \cdot[1-\beta(a)] \cdot \boldsymbol{\pi}_{1} \geq d$, always holds, the problem is trivial, the machine does not use subcontracting and inventory and backlog are negligible. Consequently, subcontracting is used to receive additional capacity when the machine is no longer capable to fulfill product demand by its own.

The decision variables of the control model are the production rates $\left(u_{1}, u_{2}\right)$ for the manufacturing system and subcontracting respectively, the third decision variable is the overhaul rate $\omega_{o}(\cdot)=\mathrm{q}_{13}$. As mentioned previously, major overhaul involves the realization of a perfect maintenance, which mitigates completely the effects of deterioration, and restoring the machine to as-good-as-new conditions. In our formulation, $\omega_{o}$ defines a decision variable that properly controls the transition to overhaul mode, with the constraint $\omega_{\min } \leq \omega_{o}(\cdot) \leq \omega_{\max }$. The reciprocal of $\omega_{\min }$ and $\omega_{\max }$ denotes the minimum and maximum delays before the machine is sent for overhaul.

Furthermore, it follows that for each $\alpha \in \Omega$, with $\xi(t)=\alpha$, the set of the feasible control policies $\Gamma(\alpha)$, including the decision variables $\left(u_{1}, u_{2}, \omega_{o}\right)$, is given by:

$$
\begin{aligned}
& \Gamma(\alpha)= \\
& \left\{\left(u_{1}(\mathrm{a}, \cdot), u_{2}(\mathrm{a}, \cdot), \omega_{o}(\mathrm{a}, \cdot)\right) \in R^{3},\right. \\
& \left.0 \leq u_{1}(\mathrm{a}, \cdot) \leq \mathrm{u}_{1}^{\max }, \quad 0 \leq u_{2}(\mathrm{a}, \cdot) \leq \mathrm{u}_{2}^{\max }, \quad \omega_{\min } \leq \omega_{o}(\mathrm{a}, \cdot) \leq \omega_{\max }\right\}
\end{aligned}
$$

One important remark is that the production cost of the manufacturing system is assumed to be proportional to its production rate $\left(C_{M 1} \cdot u_{1}\right)$, while the cost of the subcontracting option $C_{M 2}$, is assumed to be much higher $0<C_{M 1} \ll C_{M 2}$. At any instant of time $t$, the state of the system is characterized by the state variables $(\xi(t), x(t), \mathrm{a}(t))$, entailing an hybrid condition, with discrete $\xi(t)$ and continuous $(x(t), \mathrm{a}(t))$ components. With such problem, it is usually convenient to define the running cost of the model $\varphi^{\alpha}(\cdot)$ in state $\alpha \in \Omega$, as follows:

$$
\varphi^{\alpha}\left(\alpha, x, a, u_{1}, u_{2}, \omega_{o}\right)=
$$




$$
c^{+} x^{+}+c^{-} x^{-}+C_{M 1} \cdot u_{1}(t)+C_{M 2} \cdot u_{2}(t)+c_{d} \cdot \beta(a) \cdot u_{1}(t)+c^{\alpha}, \quad \forall \alpha \in \Omega
$$

with:

$$
\begin{gathered}
x^{+}=\max (0, x) \\
x^{-}=\max (-x, 0) \\
c^{\alpha}=C_{r} \cdot \operatorname{Ind}\{\theta(t)=2\}+C_{o} \cdot \operatorname{Ind}\{\theta(t)=3\} \\
\operatorname{Ind}\{\theta(t)=\alpha\}= \begin{cases}1 & \text { if } \theta(t)=\alpha \\
0 & \text { otherwise }\end{cases}
\end{gathered}
$$

where $c^{+}$and $c^{-}$are the inventory and backlog cost, respectively; $C_{M 1}$ is the production cost of the manufacturing system; $C_{M 2}$ refers to the cost of subcontracting, and $c_{d}$ denotes the cost due to the additional inspection and disposal of defective parts. In addition, $C_{r}$ defines the repair cost, and $C_{o}$ denotes the overhaul cost. The value function of the planning problem is given by:

$$
\begin{aligned}
& v(\alpha, x, a)= \\
& \quad \inf _{\left(u_{1}, u_{2}, \omega_{o}\right) \in \Gamma(\alpha)} E\left[\int_{0}^{\infty} e^{-\rho t} \varphi^{\alpha}(\cdot) d t \mid \quad \xi(0)=\alpha, x(0)=x_{0}, a(0)=a_{0}\right], \forall \alpha \in \Omega, x \in R, a \in N
\end{aligned}
$$

where $\rho$ is the discount rate and $E\left[\cdot \mid \alpha, x_{0}, a_{0}\right]$ symbolize the conditional expectation operator. The production, subcontracting and overhaul planning problem is the determination of the optimal policy $\left(\mathrm{u}_{1}^{*}, \mathrm{u}_{2}^{*}, \omega_{\mathrm{o}}^{*}\right)$ corresponding to the value function given by (8). In Appendix A, the value function is shown to be continuously differentiable and viscosity solution to the following Hamilton-Jacobi-Bellman (HJB) equations:

$$
\begin{aligned}
& v(\alpha, x, a)= \\
& \begin{aligned}
\inf _{\left(u_{1}, u_{2}, \omega_{o}\right) \in \Gamma(\alpha)}\{ & g\left[\alpha, x, a, u_{1}, u_{2}, \omega_{o}\right]+\frac{\partial v}{\partial x}[\alpha, x, a] \dot{x}+\frac{\partial v}{\partial a}[\alpha, x, a] \dot{a} \\
& +Q(\cdot) V[\alpha, x, \varphi(\xi, a)](\alpha)\}
\end{aligned}
\end{aligned}
$$

With

$$
\varphi(\xi, a)=\left\{\begin{array}{cc}
0 & \text { if } \xi\left(\tau^{+}\right)=1 \text { and } \xi\left(\tau^{-}\right)=3 \\
a\left(\tau^{-}\right) & \text {otherwise }
\end{array}\right.
$$

Where $\varphi(\xi, a)$ defines a reset function that describes the benefit of the overhaul in the system. Hence, when the value function is available, an optimal control policy can be obtained, as in equation (9). However, an analytical solution of equations (9) is almost impossible to obtain. They have been solved only by Akella and Kumar [1] and Bielecki and Kumar [29], for the simplest single machine and single part-type manufacturing system under specific assumptions which are not appropriate for the complex system considered in this paper. Given the importance of the considered planning problem for the manufacturing industries (as stated in Section 2), we adopted numerical methods to 
obtain the structure of the control policies. Since it is requested both for the operation of systems in real time and by researchers, in order to attempt the objectives of the production firms and to contribute in the control literature. Nevertheless, analytical solutions of the HJB equations (9) is a challenge considered unsurmountable in the literature, as noted by Olsder and Suri [30] and Wang and Gershwin [31]. Fortunately, the HJB equations have motivated a number of methods to approximate a solution. More specifically, Boukas and Haurie [32] showed that implementing the Kushner's method can solve such a problem in the context of production planning. Following the works of Boukas and Haurie [32], Dehayem et al. [20] and references therein, the numerical methods used to solve the proposed optimality conditions are presented in Appendix B.

\section{Optimal control policy}

As mentioned previously the purpose of this study is to analyze simultaneously the production, subcontracting and overhaul strategies for a manufacturing system subject to deterioration by utilizing a stochastic optimal control formulation. To this end, we conduct a numerical example to solve the discrete version of the HJB equations (9). A finite grid $G_{x \mathrm{a}}$ is needed to define the computational domain for the state variables $(x, a)$ :

$G_{x \mathrm{a}}=\{(\mathrm{x}, \mathrm{a}):-5 \leq \mathrm{x} \leq 10, \quad 0 \leq \mathrm{a} \leq 100\}$

We shall display the control policy of the simultaneous production, overhaul and subcontracting through numerical results. Table 1 shows the set of parameters needed in the numerical example.

\begin{tabular}{|c|c|c|c|c|c|}
\hline Parameter: & $\begin{array}{c}\mathrm{c}+ \\
\text { (\$/products/time units) } \\
1\end{array}$ & $\begin{array}{c}\mathrm{c}- \\
\text { (\$/products/time units) } \\
250\end{array}$ & $\begin{array}{c}\mathrm{c}_{\mathrm{r}} \\
(\$ / \text { repair }) \\
4 \\
4\end{array}$ & $\begin{array}{c}\mathrm{c}_{\mathrm{o}} \\
\text { (\$/overhaul) } \\
8\end{array}$ & $\begin{array}{c}\mathrm{C}_{\mathrm{M} 1} \\
\text { (\$/product) } \\
4 \\
\end{array}$ \\
\hline Parameter: & $\begin{array}{c}\mathrm{C}_{\mathrm{M} 2} \\
\text { (\$/product) }\end{array}$ & $\begin{array}{c}\mathrm{c}_{\mathrm{d}} \\
\text { (\$product) }\end{array}$ & $h_{\mathrm{x}}$ & $h_{\mathrm{a}}$ & $\theta$ \\
\hline Value: & 40 & 10 & 0.5 & 0.5 & 0.4 \\
\hline Parameter: & $\mathrm{q}_{12}(1 /$ time units $)$ & $\mathrm{q}_{21}(1 /$ time units $)$ & $\omega_{\text {(1/time units) }}^{\omega_{\min }}$ & $\begin{array}{c}\omega_{\max } \\
\text { (1/time units) }\end{array}$ & $\begin{array}{c}\mathrm{u}_{1}^{\max } \\
\text { (product/time } \\
\text { units) }\end{array}$ \\
\hline Value: & 0.4 & 5 & $10^{-6}$ & 20 & 9 \\
\hline Parameter: & $\mathrm{q}_{31}$ (1/time units) & $\begin{array}{c}d \\
\text { (products/time units) }\end{array}$ & $\begin{array}{c}\mathrm{u}_{2}^{\max } \\
\text { (product/time units) }\end{array}$ & $\rho$ & \\
\hline Value: & 4 & 4 & 20 & 0.9 & \\
\hline
\end{tabular}

Table 1. Parameters for the numerical example

Additionally, we define $k_{1}=0.1$ for Equation (3) and $k_{2}=15 \times 10^{-6.2}, b_{1}=0.01$ and $b_{2}=0.99$ for Equation (1). The reciprocal 1/ $\omega_{\max }$ denotes the delay for sending the machine to overhaul activities; in this case, for $\omega_{\max }$, this delay is insignificant, while for the case of $\omega_{\min }$ the delay $\left(1 / \omega_{\min }\right)$ will take such a long time $\left(10^{6}\right.$ times units), that it can be assumed that the overhaul will not be conducted. With these values, the capacity 
constraint (5) is satisfied. In the remainder of this section, we detail the production, subcontracting and overhaul policies, and also we discuss how they are implemented simultaneously in a joint control policy.

\subsection{Production policy}

We proceed by first examining the obtained production policy $u_{1}^{*}(\alpha, x, a)$, which indicates the production rate applied in operational mode $\alpha=1$, for any stock level $x$ and age $a$ of the manufacturing system, as illustrated in Figure 3. Such policy divides the plan $(x, a)$ into three regions, where the production rate is set to three possible options; $\mathrm{u}_{1}^{\max }, d /[1-\beta(a)]$, and 0 , respectively. In Region I the stock level surpasses the production threshold, and then the machine is not operational. In Region II, the stock level is equal to the production threshold, where the machine first operates at rate $d /[1-\beta(a)]$ from age $a=0$ to $a=60$, and then after $a=60$, it works at the maximum rate due to its high level of deterioration. When the stock level is below the production threshold, the machine always produces at the maximum rate, as denoted in Region III. Moreover, in Figure 3, we can observe that the trend in the production threshold decreases as the age of the manufacturing system increases, until a point where the machine no longer operates. This reduction clearly reflects the effect of the quality deterioration on the production policy.

We analyze the trace of the production policy from three different perspectives to facilitate its characterization, as presented in Figure 4. For the plan $\left(u_{1}, a\right)$, if we set the value of the stock level to $x=-5$, the dotted line in Figure 4a represents the production rate as observed in Figure 3. However, at considering the quality deterioration and Equation (5), such a boundary actually decreases progressively by a factor $[1-\beta(a)]$. $\boldsymbol{\pi}_{1}$, until it reaches the point $B$, where the manufacturing system stops functioning, because it is no longer profitable to operate the machine due to the high level of defectives that it produces. 


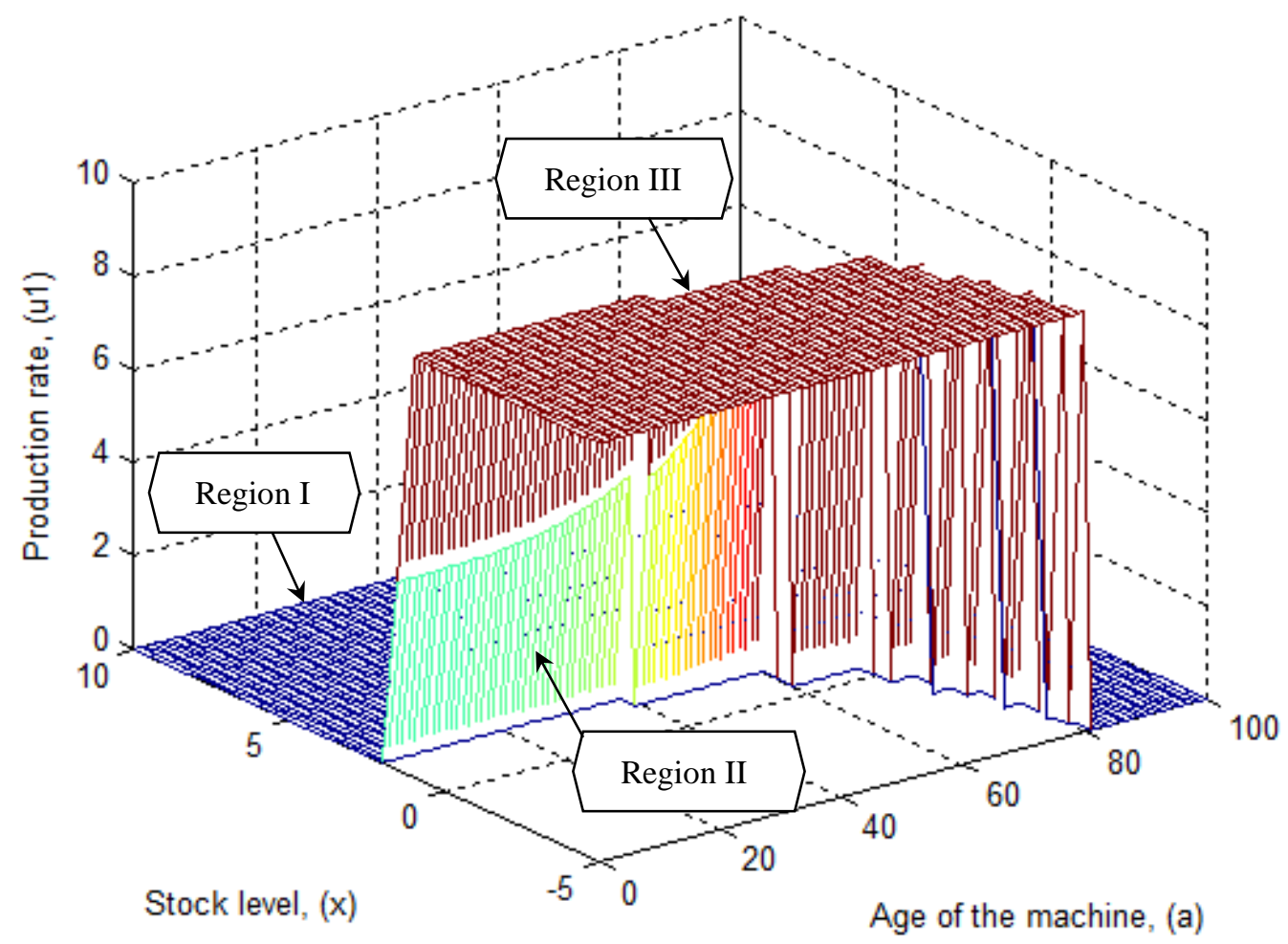

Figure 3: Production rate for the manufacturing system

The solid line in Figure 4a denotes the production rate for the manufacturing system, considering the progressive reduction caused by deterioration. We complement the results with Figure $4 \mathrm{~b}$, where we use the plan $\left(u_{1}, a\right)$ when the stock level is equal to the production threshold $x=Z_{1}(a)$. In this instance, we can clearly observe how the production rate $u_{1}^{*}$ increases by an amount of $1 /[1-\beta(a)]$ until it reaches $u_{1}^{\max }$ at point $A$, and is finally set to $u_{1}^{*}=0$ at point $B$. Moreover, we use Figure $4 \mathrm{c}$ to display the trace of the production policy in the plan $(x, a)$, which helps us identify two zones:

- Zone $A_{u 1}$ : the manufacturing system operates at maximum rate to reach the production threshold $Z_{1}^{*}(\cdot)$.

- Zone $B_{u 1}$ : the optimal production policy recommends that the manufacturing system should not be operated. 


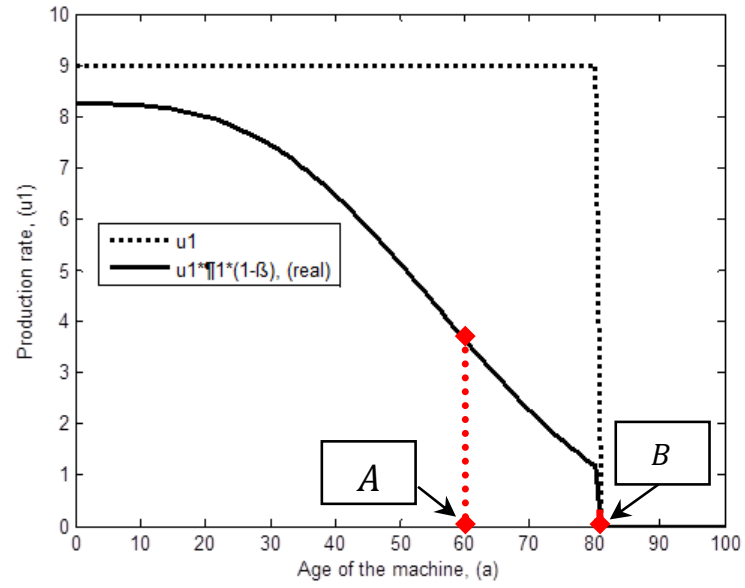

a) Plan $\left(u_{1}, a\right)$ and $x<Z_{1}(a)$

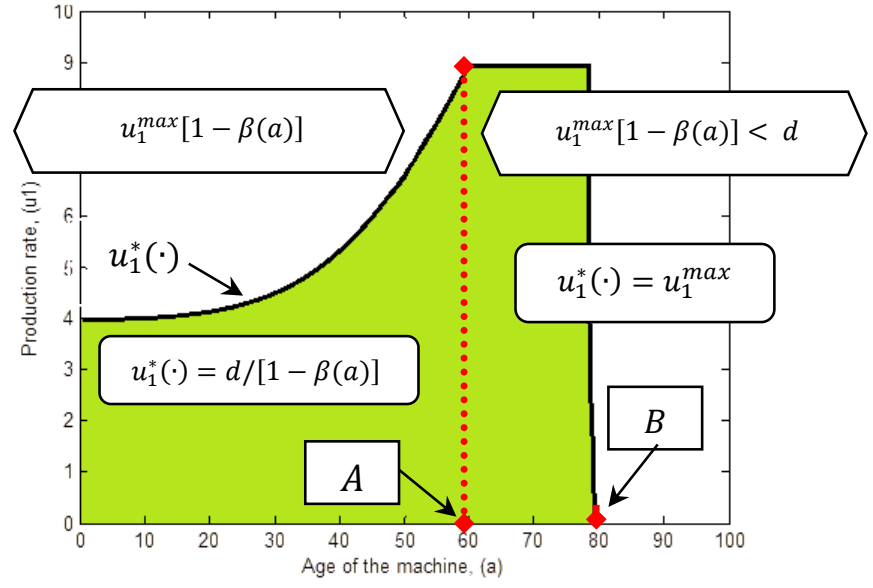

b) Plan $\left(u_{1}, a\right)$ and $x=Z_{1}(a)$

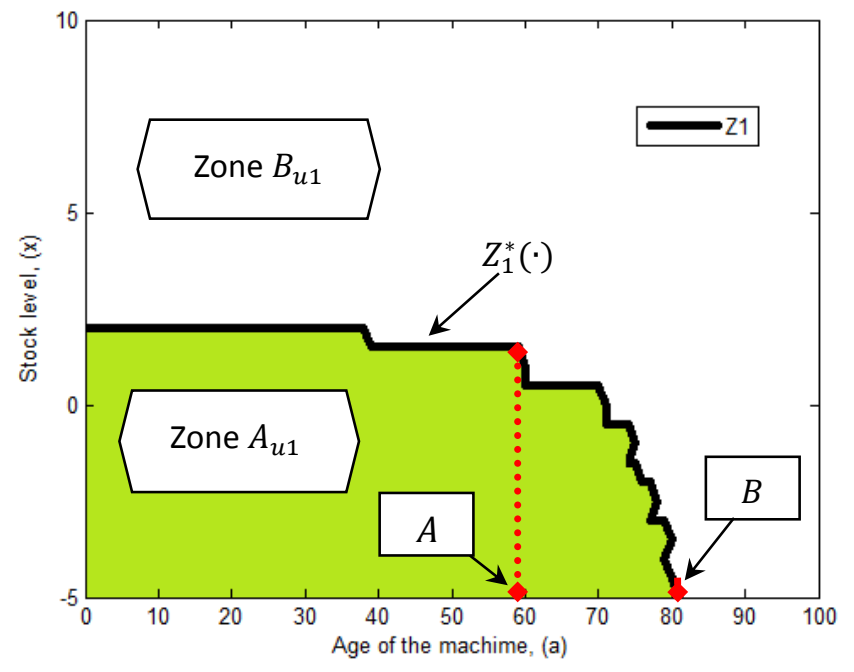

c) Plan $(x, a)$

Figure 4: Production trace

In a practical sense, the progressive reduction observed in the production threshold in Figure $4 \mathrm{c}$ can be explained because as the rate of defectives increases with the age, this also increases the total cost, thus making it more expensive to operate the manufacturing system. Therefore, after point $B$, it is no longer profitable to continue its operation. Based on these results, it can be stated that the production policy follows an age-dependent hedging point policy, which is determined in three intervals: $a \leq A, A<a<B$ and $a \geq B$; point $A$ indicates the age when $u_{1}^{\max }=d /[1-\beta(a)]$, at which subcontracting is required. The production policy is thus defined as below:

- For the case where $a \leq A$, we have $u_{1}^{\max } \geq d /[1-\beta(a)]$, hence the production policy is:

$$
u_{1}^{*}(1, x, a)=\left\{\begin{array}{cl}
u_{1}^{\max } & \text { if } x<Z_{1}(a) \\
d /[1-\beta(a)] & \text { if } x=Z_{1}(a) \\
0 & \text { if } x>Z_{1}(a)
\end{array}\right.
$$


- For the case where $A<a \leq B$, the machine is no longer capable of satisfying the product demand on its own because $u_{1}^{\max }<d /[1-\beta(a)]$; the production policy is therefore:

$$
u_{1}^{*}(1, x, a)=\left\{\begin{array}{c}
u_{1}^{\max } \text { if } x \leq Z_{1}(a) \\
0 \quad \text { otherwise }
\end{array}\right.
$$

- For the case where $a>B$, it is not profitable to continue operating the manufacturing system, which must then be stopped:

$$
u_{1}^{*}(1, x, a)=\{0 \quad \text { in any case }
$$

In the production policy $Z_{1}(a)$ represents the trace function that delimits the zone for the optimal production threshold at the operational mode as presented in Figure 4c. From the results obtained, it is clear that the production policy denotes multiple threshold levels influenced by the deterioration of the quality of the parts produced.

\subsection{Subcontracting policy}

The next step in our analysis covers the subcontracting policy. From the numerical results, we observe that this policy is controlled, as presented in Figure 5. This policy divides the plan $(x, a)$ in three regions. The first one is defined when the age of the manufacturing system is below a certain limit, and where subcontracting is not required (i.e., before age 60 in Figure 5, Region I). Then in Region II, the machine has deteriorated, and the subcontractor works first on the difference of demand that the machine is not capable of satisfying $\left(d-u_{1}^{\max } \cdot[1-\beta(a)]\right)$, and then it works at the demand rate. In Region III, the subcontractor works at its maximum rate $u_{2}^{\max }$.

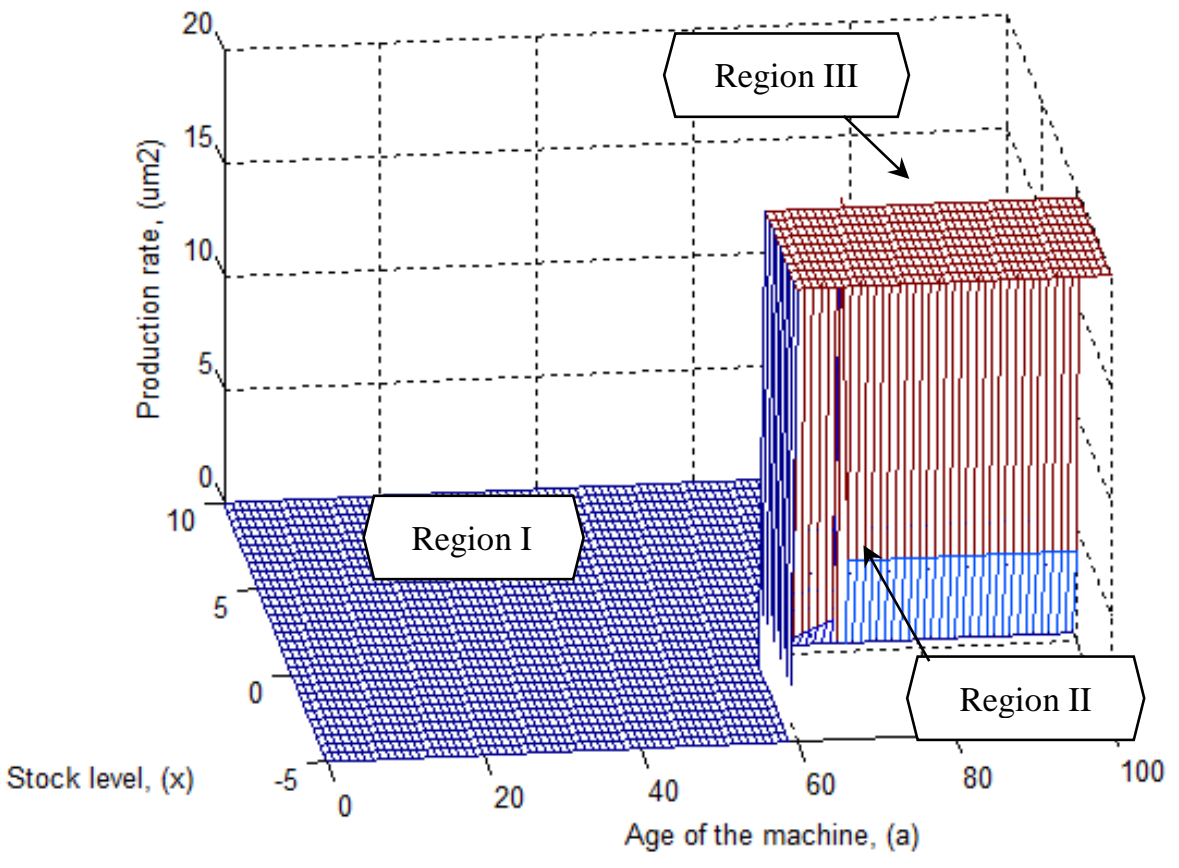

Figure 5: Subcontracting policy 
To better illustrate the subcontracting policy, we need to consider it simultaneously with the production policy of the manufacturing system. We present the related traces for the plan $\left(u_{2}, a\right)$ in Figure 6a and Figure 6b, and for the plan $(x, a)$ in Figure 6c. If we observe the plan $\left(u_{1}, a\right)$, and if the stock level is $x<0$, the subcontractor operates at the maximum rate $u_{2}^{\max }$ from point $A$ to $a=100$ (age limit of the numerical example), as presented in Figure 6a. When the stock level is $x=0$, we observe in Figure $6 \mathrm{~b}$ that from age $a=60$ to $a=70$, the subcontractor first produces the difference $d-u_{1}^{\max }$. $[1-\beta(a)]$ that the machine is not capable of satisfying due to its high level of deterioration, then when $\quad a>70$ the subcontractor produces at the demand rate. We complement the results with Figure 6c to display the trace of the production and subcontracting policies in the plan $(x, a)$, which helps us identify two zones:

- Zone $A_{u 2}$ : The rate of defectives $\beta$ is low and the manufacturing system has enough capacity to satisfy the demand, hence subcontracting is not needed.

- Zone $B_{u 2}$ : The manufacturing system is no longer capable of satisfying the products demand on its own, the age of the manufacturing system has reached point $A$, and so subcontracting is needed to fulfill the demand.

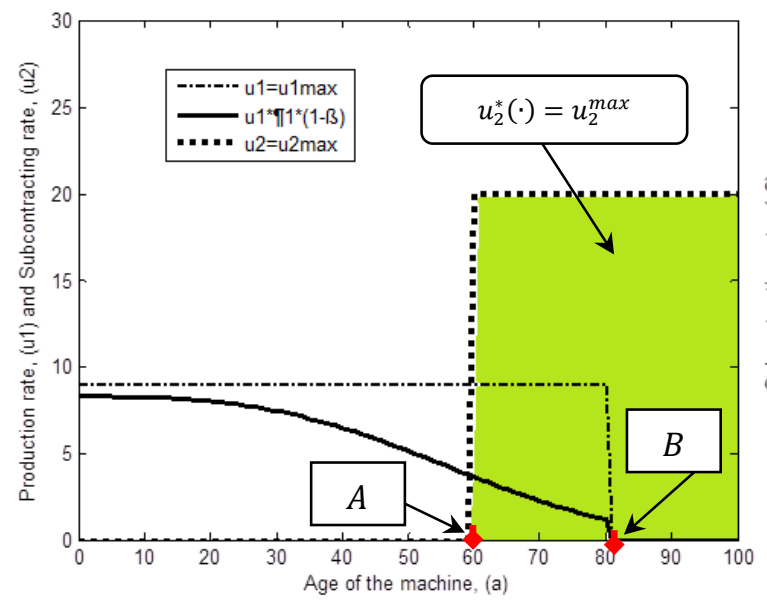

a) In plan $\left(u_{1}, a\right)$ and $x<0$

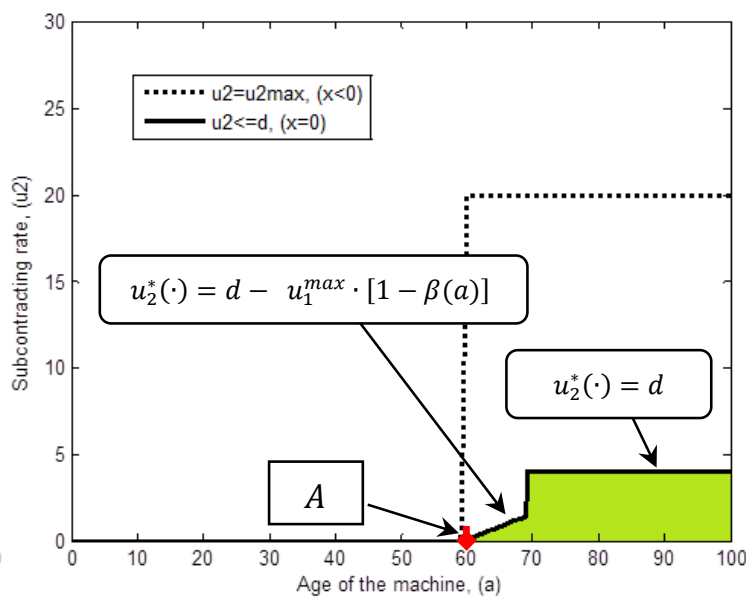

b) In plan $\left(u_{1}, a\right)$ and $x=0$

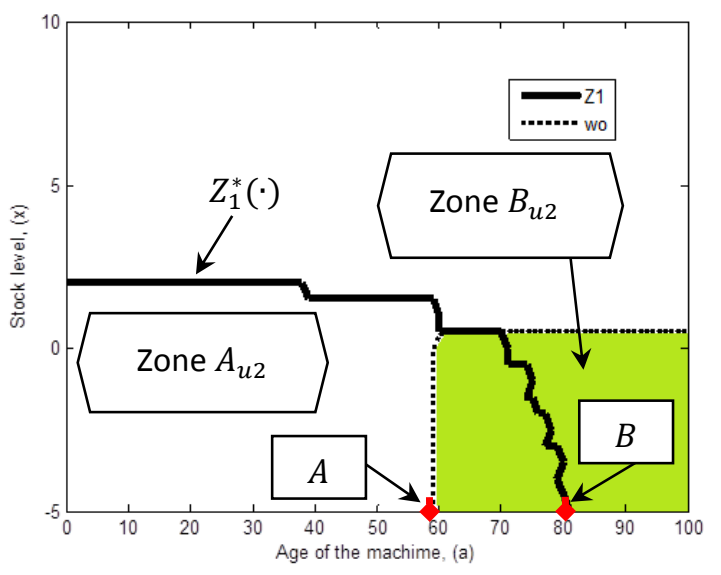

c) In plan $(x, a)$

Figure 6: Production and sub-contracting trace 
The underlying pattern of the subcontracting policy stems from the fact that the cost of subcontracting $C_{M 2}$ is higher than the production cost of the manufacturing system $C_{M 1}$. Subcontracting is only triggered when the manufacturing system is not capable of satisfying the demand, and when the stock level is lower than zero, since subcontracting is free of disruptions (such as defectives and failures). Thus, subcontracting is conducted at a rate $u_{2}^{*}$ as indicated in the following two intervals:

- For the case where $a \leq A$, we may recall that $u_{1}^{\max } \geq d /[1-\beta(a)]$, and hence the subcontracting policy is:

$$
u_{2}^{*}(1, x, a)=\{0 \quad \text { in any case }
$$

- For the case where $A<a$, we have $u_{1}^{\max }<d /[1-\beta(a)]$, and subcontracting is required, as follows:

$$
u_{2}^{*}(1, x, a)=\left\{\begin{array}{cc}
u_{2}^{\max } & \text { if } x<0 \\
d-u_{1}^{\max } \cdot[1-\beta(a)] & \text { if } x=0 \text { and } Z_{1}^{*}(\cdot)=0 \\
d & \text { if } x=0 \text { and } Z_{1}^{*}(\cdot)<0 \\
0 & \text { otherwise }
\end{array}\right.
$$

From the subcontracting policy obtained, we can clearly observe the dependence of the level of deterioration of the manufacturing system on the decision to trigger subcontracting activities; we note that with more deterioration come more defectives, and the age of the machine represents a control parameter for this policy.

\subsection{Overhaul policy}

We concentrate now on the characterization of the optimal overhaul. From the results of the numerical example, we obtain Figure 7, which illustrates when to conduct overhaul activities; we observe that an overhaul is only conducted when the level of deterioration of the manufacturing system (in other words, its age) has reached a certain limit $S A$. Since the cost of a major overhaul is high, the manufacturing system must have deteriorated enough to justify its realization. The pattern of the overhaul policy divides the plane $(\mathrm{x}, a)$ into two regions, described as follows:

- Zone $A_{o}$ : The recommendation is to perform overhaul activities, because the amount of defectives justifies the cost for this type of maintenance.

- Zone $B_{o}$ : In this zone of the plane, an overhaul is not recommended, and it is more profitable to continue operating the manufacturing system.

Considering the production threshold, we can additionally determine:

- Zone $A_{o}^{\prime}$ : Defined with the intersection of the production and overhaul policies, because the stock level is always restricted by the production threshold $Z_{1}^{*}(\cdot)$, and only a section of the overhaul zone $A_{o}$ is active. 
Additionally, when the manufacturing system has stopped its operation (after point $B$ ), it is sent automatically to overhaul maintenance to restore the machine to initial conditions.

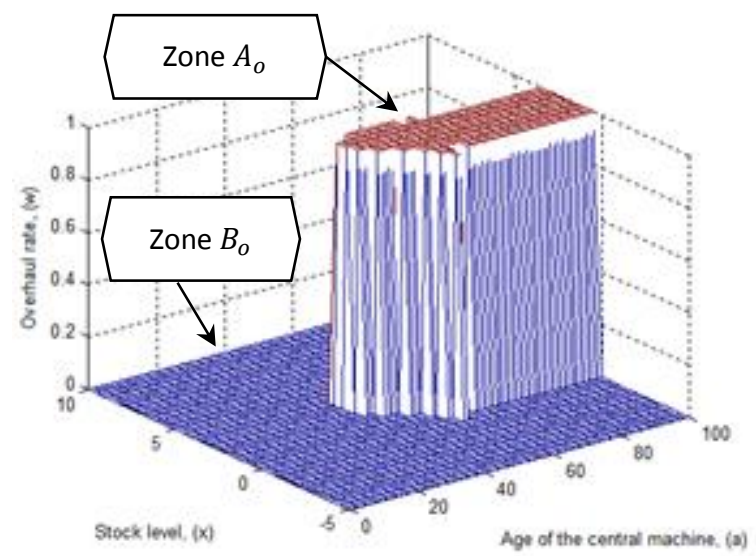

Figure 7: Overhaul policy

The logic behind the results obtained indicates that overhaul activities are conducted at a rate $\omega_{o}^{*}(\cdot)$ given by the following equation:

$$
\begin{aligned}
& \omega_{o}^{*}(1, x, \mathrm{a})= \\
& \begin{cases}\omega_{\max } & \text { if }\{[a(t)>S A \text { and } x(t) \geq s(t)] \text { or }[\mathrm{a}(t) \geq B]\} \\
\omega_{\min } & \text { otherwhise }\end{cases}
\end{aligned}
$$

where $s(\cdot)$ is the boundary of the feasible zone $A_{o}^{\prime}$. Equation (17) leads to the observation that the realization of overhaul activities involves two conditions; first, the manufacturing system is send for an overhaul when the machine has deteriorated enough (in this case, the age of the machine has reached a certain level defined by $S A$ ) to justify the high cost of an overhaul. Moreover, a certain amount of stock (defined by $s(t)$ ) is needed to compensate for the lack of product while the machine is sent for overhaul. The second condition refers to the case where the manufacturing system has deteriorated so much (its age has surpassed point $B$ ) that it produces a lot of defectives, and so the machine is sent immediately for overhaul, while subcontracting satisfies the entire product demand.

\subsection{Joint control policy}

In this section, we clarify how the obtained control policy operates simultaneously in our system. Recall that Equations (12)-(17) characterize the production, subcontracting and overhaul strategies. However, since such policies are inter-related, we display them simultaneously in Figure 8 to facilitate the analysis. At considering the policies jointly, the control parameters involved are $Z_{1}^{*}(\cdot)$ and point $B$ for the production rate of the manufacturing system, points $(A, B)$ for subcontracting, and points $S A$ and $s(t)$ for the overhaul policy, as presented in Figure 8. 


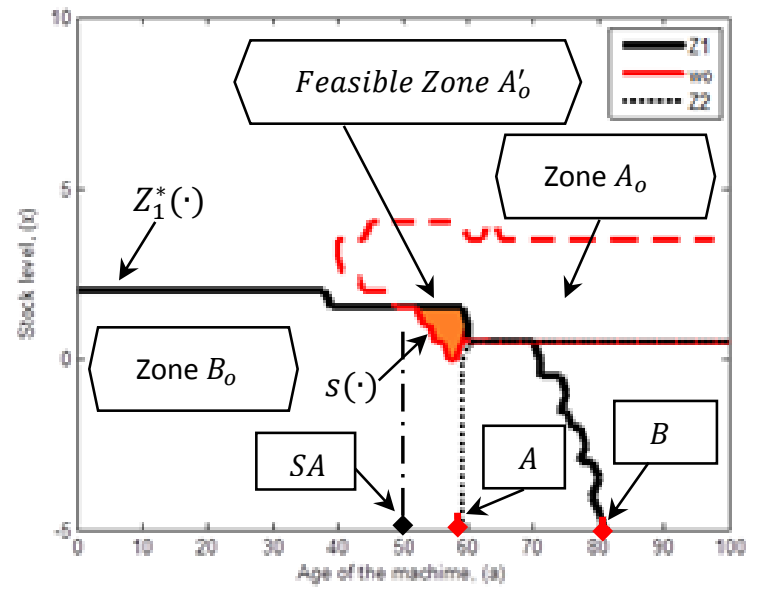

Figure 8: Joint control policy

The main observation in Figure 8, is that the overhaul trace is limited by the production boundary, because the stock level is always restricted by the production threshold $Z_{1}^{*}(\cdot)$. Thus, only a section of the overhaul zone $A_{o}$ is active, because the production, subcontracting and overhaul strategies are inter-related, this defines the feasible overhaul Zone $A_{o}^{\prime}$. Physically, such joint control policy implies that the manufacturing system $\left(\mathrm{M}_{1}\right)$ begins by producing all the product demand, then the machine gradually deteriorates, reducing its production capacity. When the age of the machine reaches point $A$, the production capacity of the machine is so reduced that subcontracting $\left(\mathrm{M}_{2}\right)$ is needed to aid to satisfy the product demand. Since the machine continues deteriorating, the cost of in-house production is so elevated in point $B$, that the machine $\left(\mathrm{M}_{1}\right)$ stops its operation. Then overhaul is conducted to restore the age to zero, mitigating all the effects of deterioration. This sequence of events implies a cycle for the deterioration process, which reinitiates with the conduction of the overhaul. Moreover, overhaul can be conducted from age $S A$ if the stock level $x>s(\cdot)$.

\section{Sensitivity and results analysis}

In this section a set of numerical examples are examined with respect to different variations of the inventory, backlog, overhaul, defectives, production and subcontracting costs. The objective of this sensitivity analysis is to compare the effect of the variation of different cost categories and two system parameters, namely the adjustment parameter $\theta$, which allows the adjustment of the trend of the ageing process, and the transition failure rate $q_{12}$. The sensitivity analysis presents the benefits sought from the joint policy and allows us to confirm and validate its structure.

\subsection{Effect of the cost variation}

In this part of the sensitivity analysis, we aim to demonstrate the robustness of the obtained joint control policy, when we vary a number of cost parameters such as: $c^{+}, c^{-}$, 
$\mathrm{c}_{\mathrm{o}}, \mathrm{C}_{\mathrm{M} 1}, \mathrm{C}_{\mathrm{M} 2}$ and $\mathrm{c}_{\mathrm{d}}$. We develop several cost parameters configurations, derived from a basic case by changing them to a high or low level. The results of the sensitivity analysis are presented in the following subsections.

\subsubsection{Variation of the backlog and inventory cost}

Following our analysis we note that the influence of the backlog $\cos t c^{-}$on the production policy is significant, as can be seen in Figure 9, where we present the production traces for the instances $c^{-}=250,350$ and 450. In observing the results, we realize that if the backlog cost is moderate, $c^{-}=250$, the production trace is less extended on the plane $(x, a)$. If we increase the backlog cost to $c^{-}=350$, the stock level increases because the product backlog is more highly penalized, and mainly because the system utilizes more stock for protection against product shortages. The observed trend maintains if we increase the backlog cost to $c^{-}=450$, where the production threshold increases even more. Further, with higher backlog costs, we prefer to operate the machine over a longer time period to help to avoid expensive backlogs, It is for this reason that point $B$ moves to the right when the backlog cost increases. The backlog cost has an effect on the subcontracting policy, since point $B$, where the machine stops operating, moves to the right as the backlog cost increases, because in the case of backlogs both the machine and subcontracting must work simultaneously to increase the stock level. The effect of deterioration is clear on the results, since the production threshold decreases as the age of the manufacturing system increases.

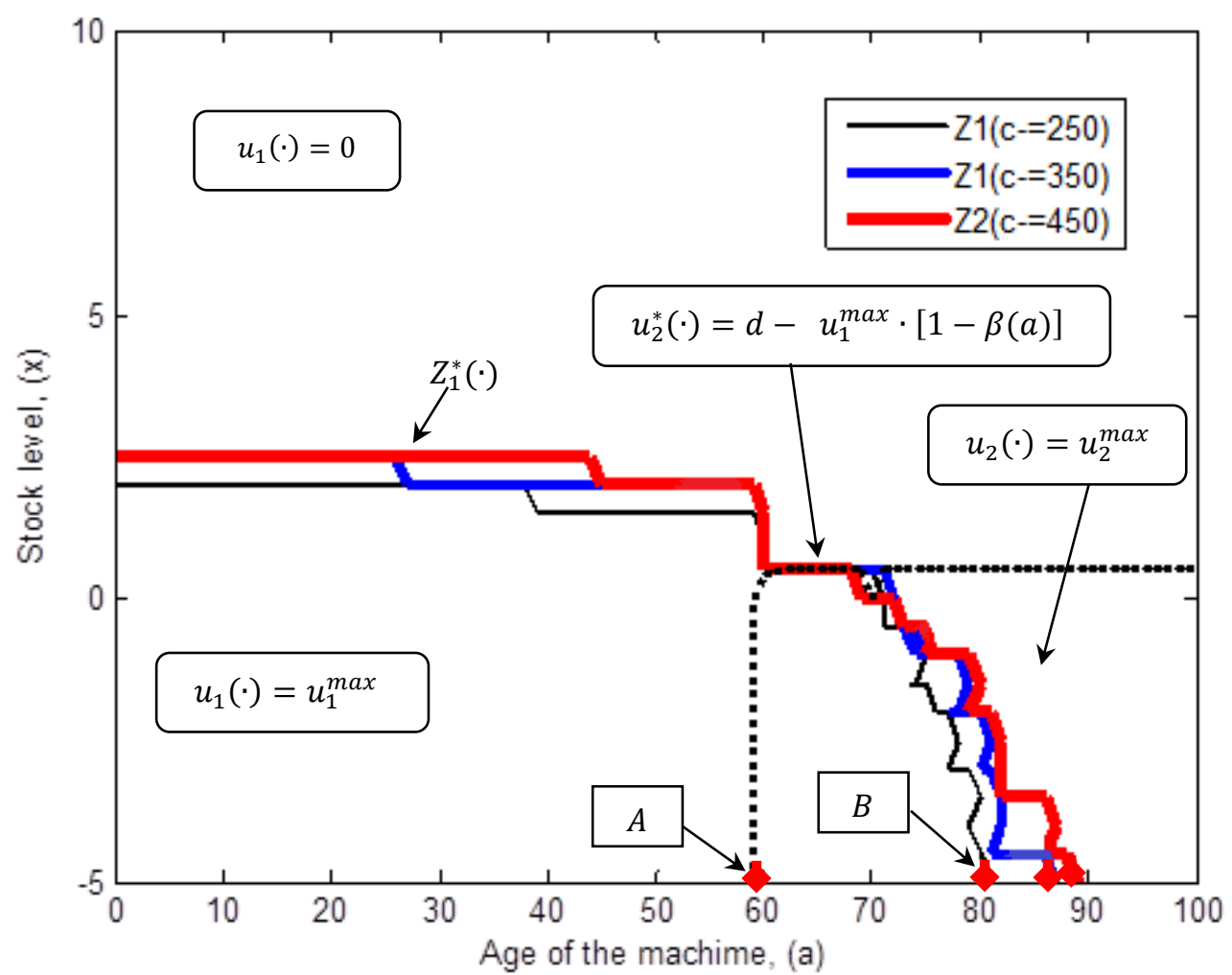

Figure 9: Influence of the backlog cost on the production and subcontracting policy 
To complement the analysis, in Figure 10, we present the overhaul trace for two different instances, $c^{-}=250$ and 450. At the lower value $c^{-}=250$, it is apparent that the feasible overhaul zone $A_{o}^{\prime}$ is the smallest of the analysis, conducting overhaul activities at age $S A=50$, and when we increase the cost to $c^{-}=450$, the zone increases further on the grid, reducing the required age to around $S A=40$. The observed pattern is derived from the fact that since the production threshold increases when the backlog cost increases, such an increase extends the feasible overhaul zone $A_{o}^{\prime}$, because when the backlog cost increases, product shortage is more penalized, and the production threshold is increased as protection. Additionally, the increase in the production threshold also increases the feasible overhaul zone because as the machine remains operational for a longer time period at $u_{1}^{\max }$ with higher backlog cost, it deteriorates more, needing extra overhaul. For the subcontracting policy, it starts at the same point $A$ in both cases. However, the machine continues operating to a higher age when the backlog cost rises, increasing point $B$ from $a=80$ to $a=90$. A sensitivity analysis was conducted on the effect of the inventory cost. We noticed that the effect of the inventory cost $\left(c^{+}\right)$on the production, overhaul and subcontracting policies is the inverse of that of the backlog cost.

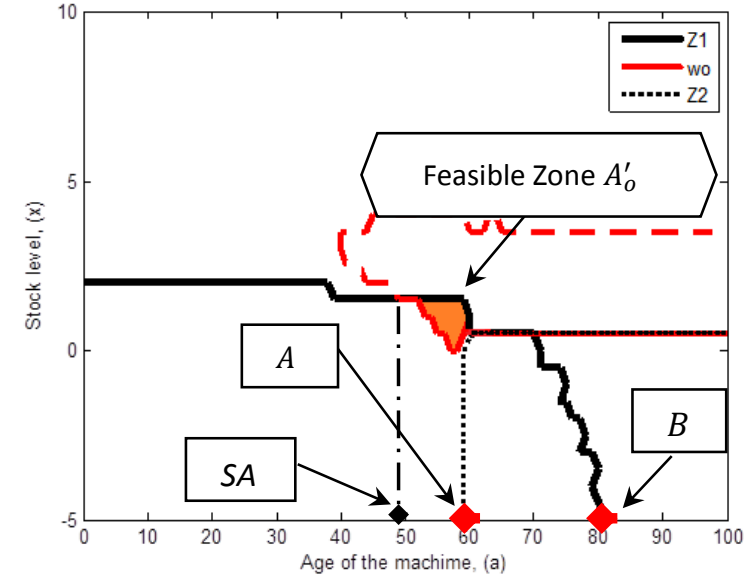

a) $c^{-}=\mathbf{2 5 0}$

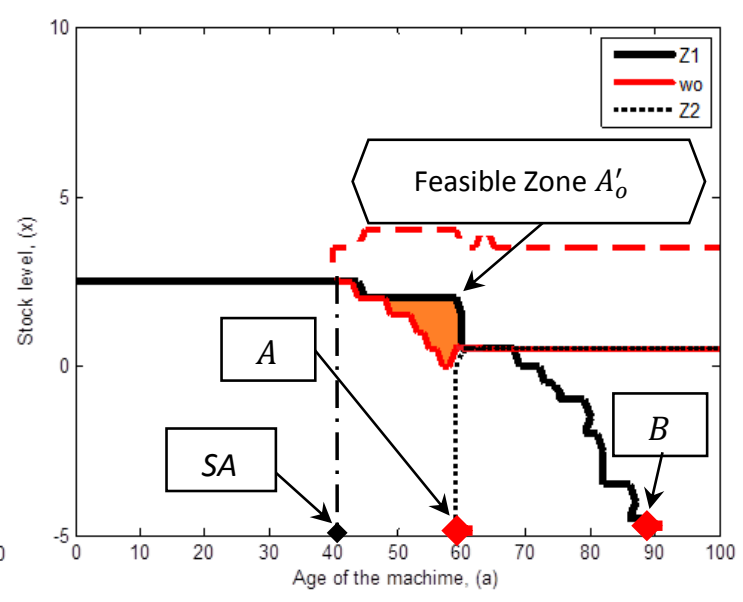

b) $c^{-}=450$

Figure 10: Influence of the backlog cost on the overhaul policy

\subsubsection{Variation of the overhaul cost}

Next, we turn our attention to the sensitivity of the overhaul cost $c_{o}$. For this stage, we examine two different scenarios using the cost values $c_{o}=5$ and 15 . The results obtained are presented in Figure 11, where we show the obtained overhaul policy. When we set the overhaul cost to the lower value of $c_{o}=5$, the result indicates that the feasible overhaul zone $A_{o}^{\prime}$ covers a greater surface area on the grid, starting overhaul activities at around age $S A=47$. If the overhaul cost is increased to $c_{o}=15$, the feasible zone $A_{o}^{\prime}$ decreases, changing the minimum required age to $S A=52$, and then requiring fewer overhaul activities, something that is normal to expect. The observed pattern in the overhaul policy implies that with higher overhaul cost, the machine must attain higher levels of deterioration, in this case the age must reach a higher level defined by $S A$, to justify the 
high cost of an overhaul. With respect to the production and subcontracting policies, we note that the overhaul cost has no effect on them, since their respective traces remain the same for both cases, because $c_{o}$ only affects the overhaul zone.

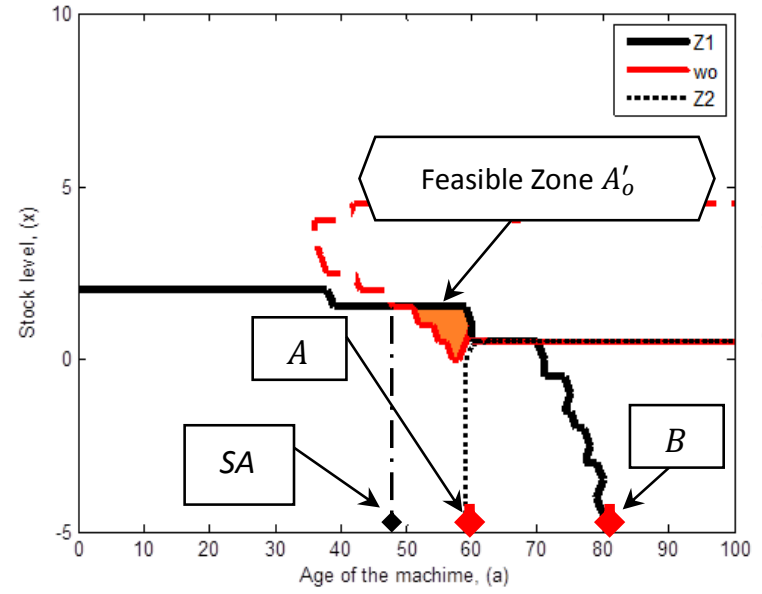

a) $c_{o}=5$

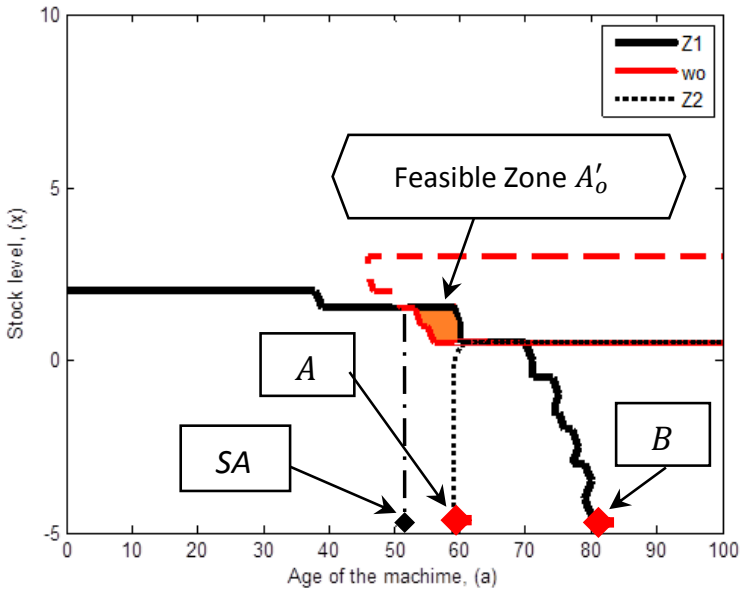

b) $c_{o}=15$

Figure 11: Influence of the overhaul cost on the overhaul policy

\subsubsection{Variation of the production and subcontracting cost}

The variation of the production $\operatorname{cost} \mathrm{C}_{\mathrm{M} 1}$ of the manufacturing system highlights the fact that it has an effect on the control policy, as can be seen in Figure 12. For this cost, we analyze two instances with cost values $C_{M 1}=2$ and 10 . At the lowest value of $C_{M 1}=2$, the production threshold of the manufacturing system is the greater of the results, since it is less expensive to operate the machine. With these higher production thresholds, it is normal to observe that the feasible overhaul zone $A_{o}^{\prime}$ also extends further on the grid. By increasing the cost value to $\mathrm{C}_{\mathrm{M} 1}=10$, we are reducing the production threshold of the machine. Because when the production cost rises, it becomes more expensive to operate the manufacturing system, and as a consequence, the production threshold decreases to just keep the bare minimum in terms of the necessary amount of products. The production cost $\mathrm{C}_{\mathrm{M} 1}$ also has an effect on the overhaul policy; the feasible overhaul zone $A_{o}^{\prime}$ contracts as the production costs rises, because with a higher production cost $\mathrm{C}_{\mathrm{M} 1}$, the machine operation is penalized more, and this reduces the production thresholds such that the machine remains operational for a shorter time, needing less overhaul. If less overhaul is required, the feasible overhaul zone reduces, from a starting age of $S A=46$ to an age of $S A=50$. The reported effect of the production cost $\mathrm{C}_{\mathrm{M} 1}$, is more clearly observed on the production and overhaul policy. The subcontracting policy is also affected; when the production cost $\mathrm{C}_{\mathrm{M} 1}$ rises, the advantage of machine $\mathrm{M} 1$ over subcontracting M2 decreases, leading to conduct more subcontracting, and reducing the age at which the machine stops operating (point $B$ ) from $a=82$ to $a=74$. Point $A$ remains in the same position, since the rate of deterioration of the machine (illustrated in Figure 2) is the same in both cases, and thus the machine is unable to satisfy demand at the same point $A$. Moreover, a sensitivity analysis was conducted on the variation of the subcontracting cost $\left(\mathrm{C}_{\mathrm{M} 2}\right)$, and we noticed that the effect of the subcontracting cost 
$\left(\mathrm{C}_{\mathrm{M} 2}\right)$ on the production, overhaul and subcontracting control policies is the opposite of that of the production cost $\left(\mathrm{C}_{\mathrm{M} 1}\right)$.

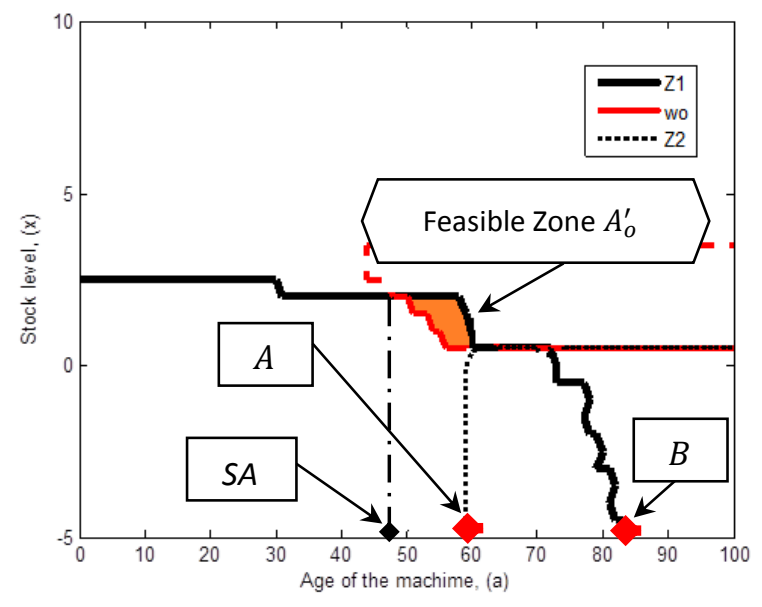

a) $C M_{1}=2$

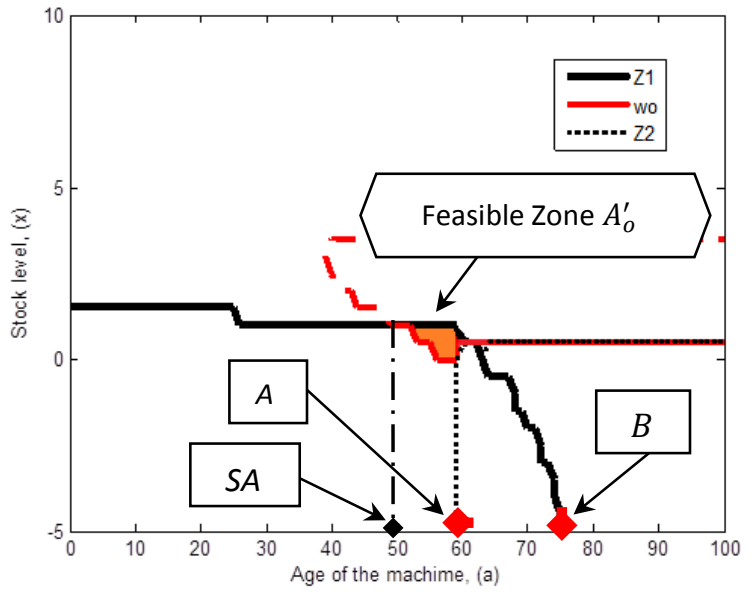

b) $C M_{1}=10$

Figure 12: Influence of the production cost of the machine on the joint policy

\subsubsection{Variation of the cost of defectives}

In this subsection, we discuss the main results of the effect of variation of the cost of defectives on the obtained control policy. We use two different cost values, $c_{d}=5$ and 15, for the analysis. The results of Figure 13 clearly show that the production trace is extented further on the grid when the cost of defectives is set to the lower value $c_{d}=5$. Nevertheles, it can be observed that if we increase the defectives cost to $c_{d}=15$, the production thresholds reduces considerably, and even the manufactuing system stops its operation at an early age, because it is more penalized defective production. To clarify matters, when we increase the defectives cost, we engange in more subcontracting, since the advantage of machine M1 over subcontracting M2 decreases, as subcontracting is free of defectives. The influence on the subcontracting policy is clear in Figure 13, since point $B$, where the manufacturing system stops producing, is reduced when we increase the defectives cost, thus acelerating its stoppage at an early age, from $a=86$ to $a=77$. Considering the overhaul policy, this actity is also influenced by the defectives cost. From the results, it follows that if we increase the defectives cost, the feasible overhaul zone $A_{o}^{\prime}$ increases, varyng the starting age from $S A=50$ to $S A=44$. This is because when the defectives cost is high, there is more emphasis on defectives, and more overhaul is conducted to improve the rate of defectives. 


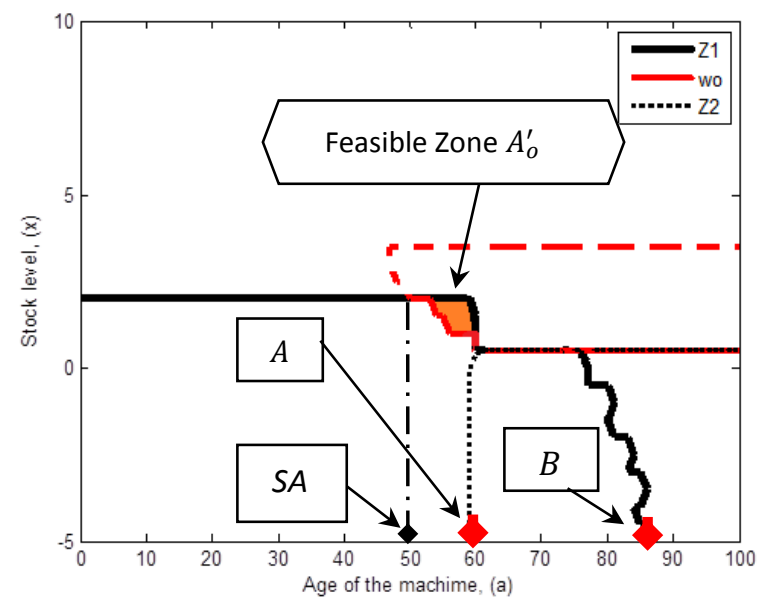

a) $c_{d}=5$

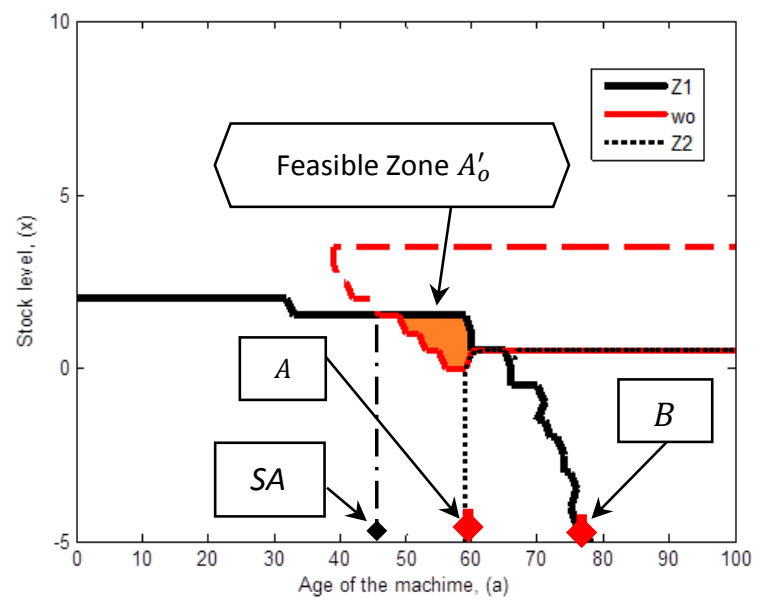

b) $c_{d}=15$

Figure 13: Influence of the defectives cost on the joint policy

\subsection{Effect of the system's parameters variation}

In the last subsection we discussed the effect of the variation of several cost parameters on the control policy. We complement our sensitivity analysis with an analysis of the variation of two system parameters, the adjustment parameter $\theta$, the failure transition rate, and the production rate of the machine $u_{1}^{\max }$. Another set of instances were thus conducted to have a better understanding of the impact of the variations of these two parameters on the joint control policy.

\subsubsection{Variation of the adjustment parameter $\theta$}

As a matter of interest, we analyze the effect of the adjustment parameter $\theta$ on the control policy obtained. As stated earlier, the parameter $\theta$ changes the pace of deterioration of the manufacturing system, by modifying the trend of the rate of defectives as a function of the age of the machine. To keep things simple, we examine two different scenarios with values $\theta=0.4$ and 1 , as presented in Figure 14. Whenever the adjustment parameter is set to the smaller value of $\theta=0.4$, the production threshold is the most extensive of the analysis. On increasing the parameter to $\theta=1$, the production threshold is reduced considerably, because at increasing $\theta$, the pace of deterioration of the manufacturing system increases considerably, leading to an earlier production of defectives, and thus stopping the machine. Based on these results, the subcontracting option is also largely affected by the parameter $\theta$, since when we increase this parameter, the machine stops earlier, because it produces defectives earlier. With this it changes the stoppage point $B$, from $a=80$ to age $a=60$. Additionally, the adjustment parameter $\theta$ modifies the point $A$, from age $a=60$ to age $a=43$. When $\theta$ increases, the machine deteriorates earlier, producing defectives, and as a result subcontracting is needed sooner to ensure demand is met with flawless units. A close examination of Figure 14 shows that the overhaul policy also is affected, because when the parameter $\theta$ is increased, the 
machine stops earlier due to its rapid deterioration, thus reducing the production trace. As a result, the feasible overhaul zone $A_{o}^{\prime}$ changes, decreasing the starting age for overhaul activities from $S A=48$ to $S A=35$. This pattern is due to the fact that with higher values of the parameter $\theta$, the machine will deteriorate earlier, and overhaul activities must be conducted sooner in order to restore the machine to as-good-as-new-conditions.

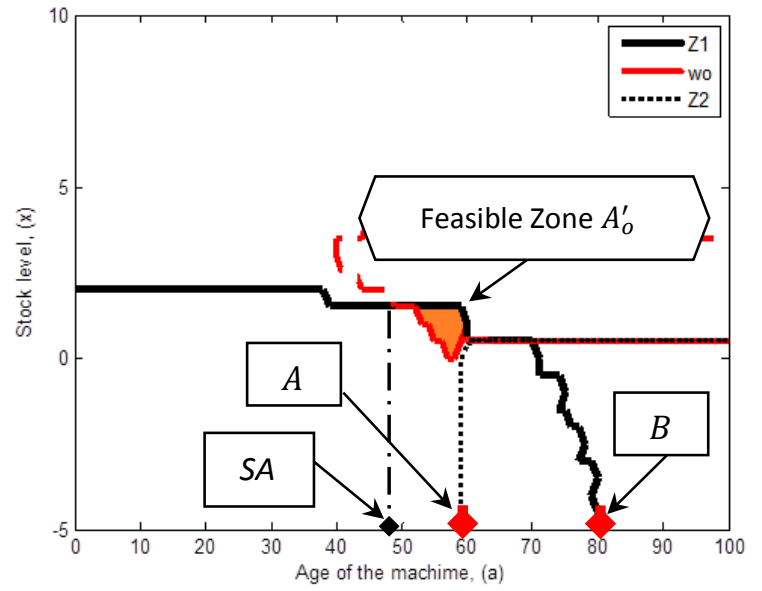

a) $\theta=0.40$

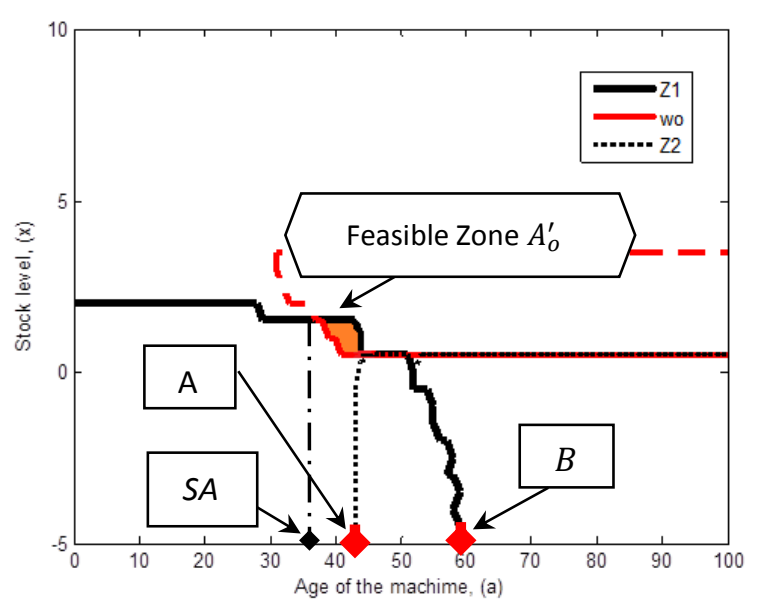

b) $\theta=1$

Figure 14: Influence of the adjustment parameter on the joint policy

\subsubsection{Variation of the failure transition rate}

We complement the analysis with a variation of the transition failure rate $q_{12}$. This transition is responsible for determining the time to failure of the manufacturing system, in addition to affecting the availability of the machine, which is reflected in its effective working time. We analyze two different cases, with values $q_{12}=0.3$ and $q_{12}=1.2$, presented in Figure 15. When we set the transition rate to $q_{12}=0.3, \pi_{1}$ reported an availability of around $94 \%$ of the operational time. With such a high availability, the production threshold was naturally the smallest in the analysis. When we increased the transition failure rate to $q_{12}=1.2$, we had an availability of $80 \%$, meaning that the production thresholds increased to protect the system against the product shortages. With respect to the subcontracting policy, we found that when we increase the transition rate $q_{12}$, subcontracting activities starts earlier, changing point $A$ from age of $a=58$ to $a=52$, because the machine earlier is no longer capable of satisfying the product demand on its own. The increment of the transition rate $q_{12}$ also modifies point $B$, which changes from age $a=82$ to $a=78$. The overhaul policy is also affected by this parameter; we observe that when increasing the transition rate $q_{12}$, we need more overhaul, and this changes the minimum required age for this activity from age $S A=48$ to $S A=37$. The reason behind these changes lies in the fact that when we increase the transition rate $q_{12}$, the production thresholds increases as protection against backlogs, causing the machine works more frequently at $u_{1}^{\max }$, and so deteriorating more. To counteract the effect of deterioration, therefore, A, B and SA change in response. 


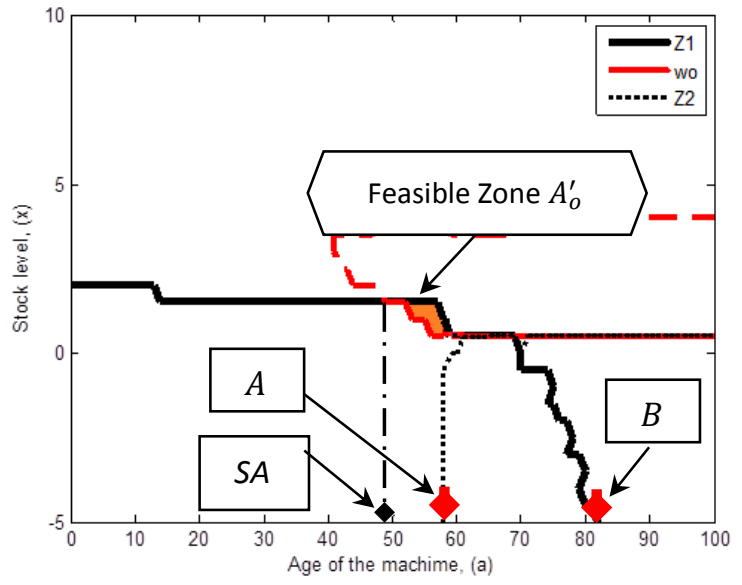

a) $q_{12}=0.3$

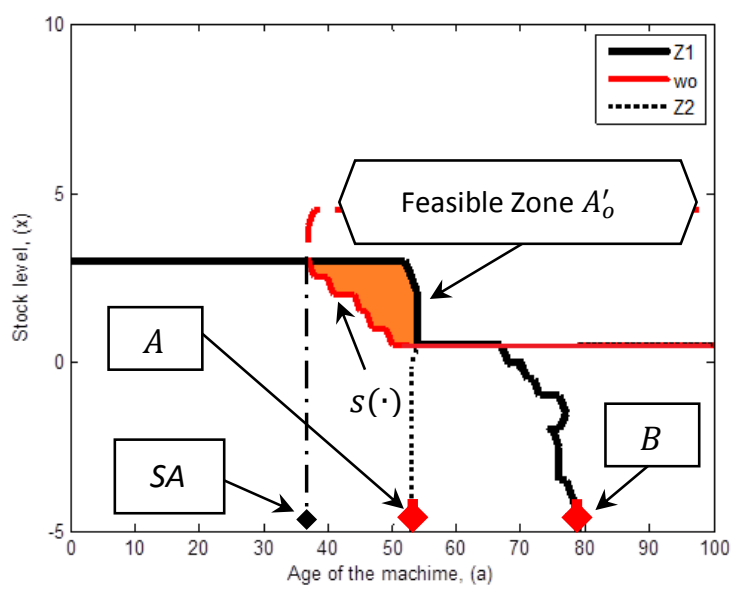

b) $q_{12}=1.2$

Figure 15: Influence of the failure transition rate on the joint policy

\subsubsection{Variation of the capacity of the machine}

The effect of the variation of the subcontracting cost was discussed in Section 6.1.3, now if we focus on the capacity of the machine we can consider the variation of the rate $u_{1}^{\max }$ as a long-term strategy for capacity planning. An increment in the capacity $u_{1}^{\max }$ incurs in a much higher cost than subcontracting, because it is implied a new machine with a superior performance to increment the rate $u_{1}^{\max }$. In the hypothetical case where we can invest unlimited resources for a new machine to increase its capacity and avoid the use of subcontracting, we analyze two cases with values $u_{1}^{\max }(\cdot)=9$ and $u_{1}^{\max }(\cdot)=15$ as presented in Figure 16. When we set the capacity of the machine to $u_{1}^{\max }(\cdot)=9$, the production, subcontracting and overhaul traces are the most extended in the grid, because we need more stock as protection against machine failures. At increasing the capacity to $u_{1}^{\max }(\cdot)=15$ with the investment of a new machine, it have several benefits, the production threshold decreases, because the system has more capacity to satisfy product demand and less stock is needed as protection against failures. Further, with $u_{1}^{\max }(\cdot)=15$ the machine works less time at its maximum rate, so it deteriorates less, thus less overhaul is needed, and this changes point $S A$, from age $a=44$ to $a=53$. Also the subcontracting trace is modified, because with more capacity, subcontracting is less needed, hence changing point $A$, from age $a=58$ to $a=68$. Based on these results, we can state that the system will be certainly more capable with a higher capacity $u_{1}^{\max }$. Nevertheless, given the investment involved, it will be considerably limited the use of this capacity strategy. In this context, subcontracting is an attractive option to satisfy product demand at a reasonable cost. 


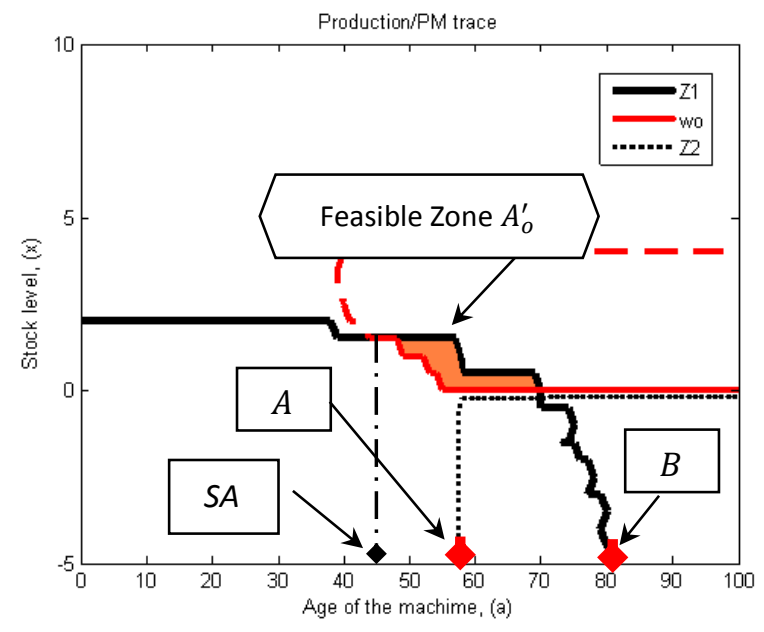

a) $u_{1}^{\max }(\cdot)=9$

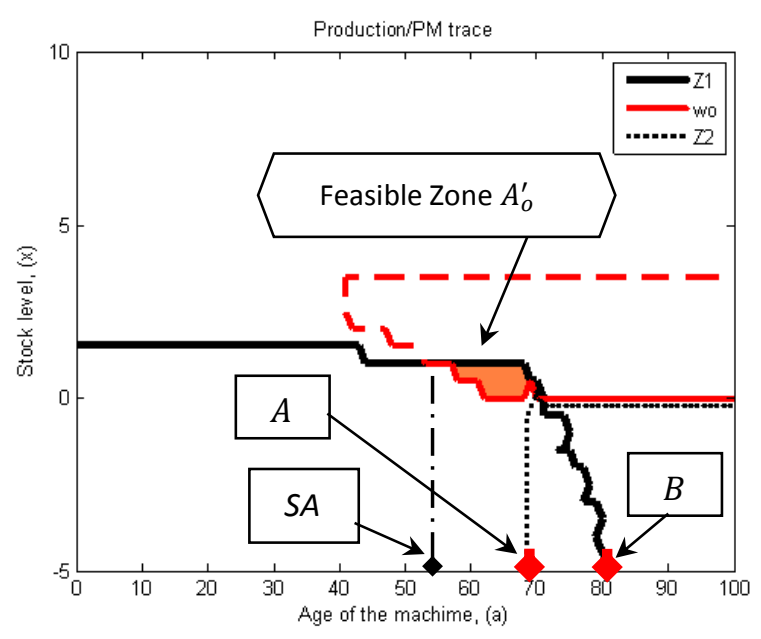

b) $u_{1}^{\max }(\cdot)=15$

Figure 16: Influence of the capacity of the machine

\section{Managerial implications}

Some implementations in real production systems of derivations of the Hedging Point Policy are the Boeing Flap Support Business Unit, reported in Gershwin [33], which focused solely in production strategies. Other efforts include the work of Dror et al. [18] for an implementation of production and subcontracting strategies for a chemical company. Managerial implications into business practice for our paper require full information about the state of the production system to implement the obtained results. The manager can control the system with our joint policy by observing the inventory and backlog level as well as the age of the machine. In light of this discussion, our policy proposes three levels; one for deciding on the safety stock, other to decide when to call subcontracting and other to indicate when to perform overhaul. The obtained dynamic programming-based policy, is reasonable for practical factory control, because it enables the system to run more smoothly and predictably, and mainly because of its ease to be implemented.

The implementation of our joint control policy is further facilitated with the use of an implementation logic chart, presented in Figure 17, which leads us through decision making. Assuming that the machine is operational and is waiting for its next failure at its age $(a)$, and with a stock level of $(x)$, the production, subcontracting and overhaul rates can be easily defined in four different intervals denoted by the points $(S A, A, B)$, as follows: $a<S A, S A \leq a<A, A \leq a<B$, and $B \leq a$, as indicated in equations (12)-(17). Hence, the implementation logic chart implies: 


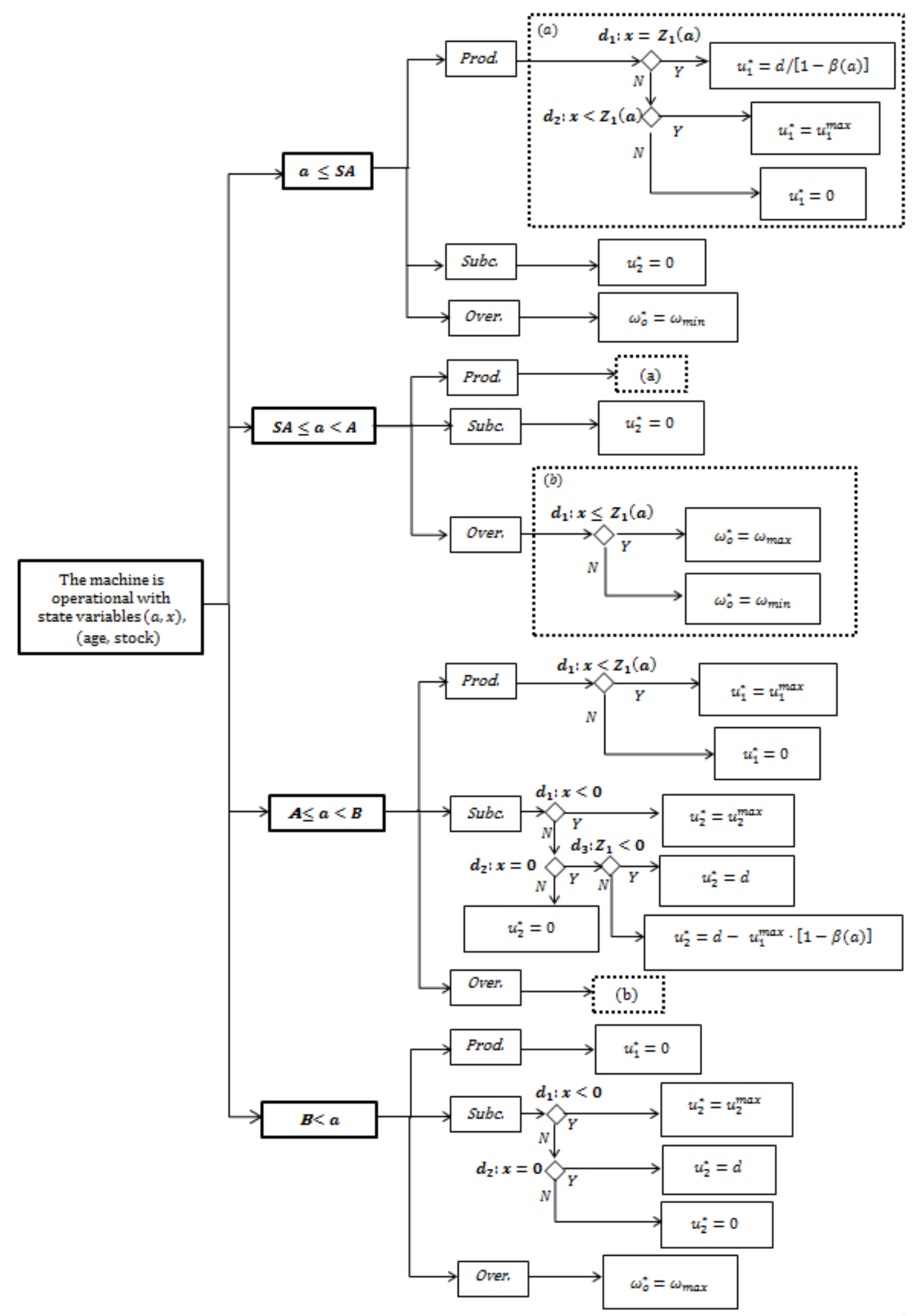

Figure 17: Implementation logic chart 
In the implementation logic chart, the production threshold $Z_{1}(a)$ must be updated continually as the age of the machine $(a)$ increases. As an illustration, we determine the control rates for seven different points located on the grid $(a, x)$ with the data of Table 1 (when $\mathrm{C}_{\mathrm{M} 1}=1$ ) as presented in Figure 18.

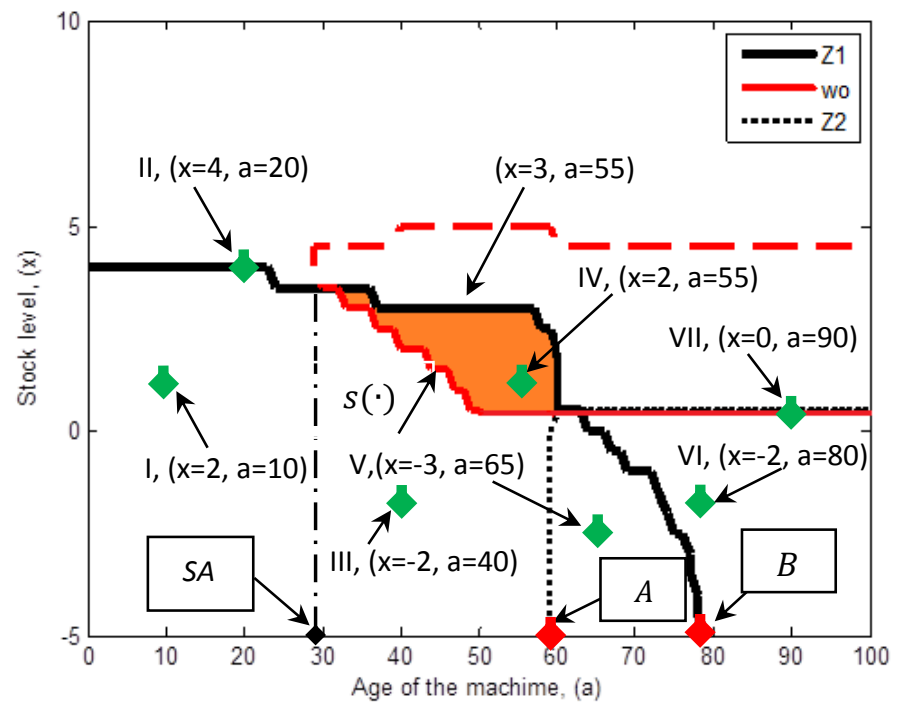

Figure 18: Implementation of the joint control policy

The different control variables are shown in Table 2 for the selected points, the logic of the policy is straightforward. For instance, in point IV, where the stock level is $x=2$, and the age is $a=55$, the production threshold is $Z_{1}=3$, overhaul will be conducted, and so $\omega_{o}^{*}(\cdot)=\omega_{\max }$, due to the stock level is within the interval $(s=0) \leq x \leq\left(Z_{1}^{*}=3\right)$. Hence, there is no production, and $u_{1}^{*}(\cdot)=0$. At this age, $a=55$, the machine has deteriorated significantly, with a rate of defectives of $\beta(55)=0.47$, (given by Equation 1 ), but it is still capable to satisfy the product demand by its own, since $u_{1}^{\max } \cdot \pi_{1} \geq d /[1-\beta(55)]$, $8.33 \geq 7.54$ therefore, subcontractors are not required.

\begin{tabular}{ccccccc}
\hline Point & $(x, a)$ & $\beta(a)$ & $Z_{1}^{*}$ & $u_{1}^{*}(\cdot)$ & $u_{2}^{*}(\cdot)$ & $\omega_{\mathrm{o}}^{*}(\cdot)$ \\
\hline I & $(2,10)$ & 0.01 & 4 & $u_{1}^{\max }=9$ & 0 & $\omega_{\min }=10^{-6}$ \\
II & $(4,20)$ & 0.05 & 4 & $d /[1-\beta(20)]=4.21$ & 0 & $\omega_{\min }=10^{-6}$ \\
III & $(-2,40)$ & 0.22 & 3 & $u_{1}^{\max }=9$ & 0 & $\omega_{\min }=10^{-6}$ \\
IV & $(2,55)$ & 0.47 & 3 & 0 & 0 & $\omega_{\max }=20$ \\
V & $(-3,65)$ & 0.65 & -1 & 0 & $u_{2}^{\max }=20$ & $\omega_{\max }=20$ \\
VI & $(-2,80)$ & 0.85 & - & 0 & $u_{2}^{\max }=20$ & $\omega_{\max }=20$ \\
VII & $(0,90)$ & 0.93 & - & 0 & $d=4$ & $\omega_{\max }=20$ \\
\hline
\end{tabular}

Table 2: Control parameters for three different instances 


\section{Conclusions}

From what has been presented, we can state that our general aim in this research was to analyze the simultaneous production planning, overhaul and subcontracting problem for the case of an unreliable machine subject to progressive deterioration, problem that is motivated from a real production context. Fundamentally, the central premise of the paper is to devise a connection between the deterioration of the machine and its quality throughput, and to allow subcontracting activities as an option to avoid backlog and fulfill product demand. The production control problem was formulated as a stochastic dynamic programming problem, which integrates the machine's history with the age of the manufacturing system, thus leading to the formulation of a Semi-Markov decision model. A numerical approach was adopted to solve the derived HJB equations of the problem, defining the control parameters of the joint policy. In general, we observe that the production threshold of the machine decreases as a function of its level of deterioration, and the conduction of overhaul and subcontracting activities, increases as an alternative to palliate the level of deterioration. A close examination of the control parameters was performed in an extensive sensitivity analysis, in which we confirmed the structure of the control policy, as well we noticed the remarkable effect of deterioration on the joint production, overhaul and subcontracting control rules. In a sense, the obtained results are quite satisfactory and point the way to further research in this domain. Future research include extending our model to the case where subcontracting is not always available due to serving other customers. Also subcontracting can be significantly influenced, when the deterioration of the machine impacts several performance indices such as the quality of the parts produced, reliability, etc., as well as their variable lead time. Another area of future research is to consider the maximum production rate of the machine as a design parameter to be optimized, since the production rate is penalized in the objective function, hence performance could be improved at optimizing the production rate. For more complex systems, the structure of the control policy developed in this paper could be the main frame for another class of future studies. For such systems, heuristic methods based on the structure of the policy could be developed. We could then consider the optimization of manufacturing systems with more general failures/repairs distributions, non-homogenous stochastic processes and large scale systems impossible to tackle only with analytical tools. The method used in such a heuristics consists of an analytical formalism, combined with discrete/continuous simulation modeling, design of experiments and response surface methodology

\section{Appendix A. Optimality conditions}

The model denoted in equations (1-10) refers to a stochastic dynamic programming problem since the minimization operation takes into account the randomness of $\alpha$. The optimality conditions of the model can be derived by the principle of optimality, if $v(\cdot, t)$ denotes a cost-to-go function at time $t$, and breaking up the integral of equation (8), we have that $v(\alpha(0), x(0), a(0), 0)=$ 


$$
\inf _{\substack{u_{1}(t), u_{2}(t), \omega_{o}(t) \\
0 \leq t \leq \infty}} E\left\{\begin{array}{l}
\int_{0}^{t} e^{-\rho t} g\left[\left(\alpha(t), x(t), a(t), u_{1}(t), u_{2}(t), \omega_{o}(t)\right] d t\right. \\
\int_{t}^{\infty} e^{-\rho t} g\left[\left(\alpha(t), x(t), a(t), u_{1}(t), u_{2}(t), \omega_{o}(t)\right] d t\right.
\end{array} \mid \alpha(0), x(0), a(0)\right\}
$$

Since the integral in the interval $[t, \infty]$ is the value function, at considering the discounted rate $\rho$, and if we perturb $t$, we can obtain the one-step counterpart of $v(\alpha(t), x(t), a(t), t)$ in the interval $[t, t+\delta t]$, with $v(\alpha(t), x(t), a(t), t)=$

$$
\inf _{\substack{u_{1}(s), u_{2}(s), \omega_{o}(s) \\
t \leq s \leq t+\delta t}} E\left\{\begin{array}{c}
\int_{t}^{t+\delta t} e^{-\rho t} g\left[\left(\alpha(t), x(t), a(t), u_{1}(t), u_{2}(t), \omega_{o}(t)\right] d s\right. \\
+ \\
\frac{1}{1+\rho \delta t} V[\alpha(t+\delta t), x(t+\delta t), a(t+\delta t), t+\delta t]
\end{array} \mid \alpha(t), x(t), a(t)\right\}
$$

where $u_{1}(s), u_{2}(s)$ and $\omega_{o}(s)$ are treated as constants in the interval $t \leq s \leq t+\delta t$. By applying the conditional expectation operator $\widetilde{E}$ (i.e., for any function $H(\alpha), \widetilde{E} H(\alpha(t+\delta t))=E\{H(\alpha(t+\delta t)) \mid \alpha(t)\})$, and considering small $\delta t$, and after some manipulations, leads to, $\rho v(\alpha(t), x(t), a(t), t)=$

$$
\inf _{u_{1}(t), u_{2}(t), \omega_{o}(t)} \tilde{E}\left\{\begin{array}{c}
g\left[\left(\alpha(t), x(t), a(t), u_{1}(t), u_{2}(t), \omega_{o}(t)\right] \delta t+\right. \\
+ \\
\frac{v[\alpha(t+\delta t), x(t+\delta t), n(t+\delta t), t+\delta t]-v[\alpha(t), x(t), n(t), t]}{\delta t}
\end{array}\right\}+o(\delta t)
$$

We can apply the full derivative of the value function, since the second term in Equation (A.3) denotes the derivative of $v(\alpha, x, a)$. Further, at expanding the expectation operator (i.e., with $\left.\widetilde{E} H(\alpha(t+\delta t))=H(\alpha(t))+\sum_{j} H(j) \lambda_{j \alpha(\mathrm{t})} \delta t+o(\delta t)\right)$, we get, $\rho v(\alpha(t), x(t), a(t), t)=$

$$
\inf _{u_{1}(t), u_{2}(t), \omega_{o}(t)}\left\{\begin{array}{c}
g\left[\left(\alpha(t), x(t), a(t), u_{1}(t), u_{2}(t), \omega_{o}(t)\right] \delta t+\right. \\
\frac{\partial v}{\partial x}[\alpha(t), x(t), a(t), t] \delta x(t)+\frac{\partial v}{\partial a}[\alpha(t), x(t), a(t), t] \delta a(t) \\
\frac{\partial v}{\partial t}[\alpha(t), x(t), a(t), t] \delta t+\sum_{\alpha^{\prime}} v\left[\alpha^{\prime}, x(t), a(t), t\right] \lambda_{\alpha^{\prime} \alpha(\mathrm{t})} \delta t
\end{array}\right\}+o(\delta t)
$$

The expectation symbol is replaced with the summation term. Furthermore, at replacing $\delta x(t)$ by $\delta x(t)=\dot{x}(t) \delta t$ and $\delta a(t)$ by $\delta a(t)=\dot{a}(t) \delta t$, move $\frac{\partial V}{\partial t}$ to the left, let $\delta t$ approach zero. After some manipulations, we have that $\rho v(\alpha, x, a, t)-\frac{\partial v}{\partial t}[\alpha, x, a, t]=$

$$
\inf _{u_{1}(t), u_{2}(t), \omega_{o}(t)}\left\{g\left[\alpha, x, a, u_{1}, u_{2}, \omega_{o}\right]+\frac{\partial v}{\partial x}[\alpha, x, a, t] \dot{x}+\frac{\partial v}{\partial a}[\alpha, x, a, t] \dot{a}+\sum_{\alpha^{\prime}} V\left[\alpha^{\prime}, x, a, t\right] \lambda_{\alpha^{\prime} \alpha}\right\}
$$

Given that none of the functions $g(\cdot), \dot{x}(\cdot)$ and $\dot{a}(\cdot)$ are functions of $t$ explicitly, and given that the time horizon is infinite and a steady-state distribution exists for $\alpha$, equation (A.5) is independent of $t$. Additionally, the summation term can be replaced by the generator $Q(\cdot)=\left\{\lambda_{\alpha \alpha^{\prime}}(\cdot)\right\}$, hence this leads to the so-called Hamilton-Jacobi-Bellman (HJB) equations given by equation (9), their importance relies on the fact that it is a necessary and 
sufficient condition for an optimum. In consequence, the HJB equations, describe the optimally conditions of the problem, thus control policies obtained from the HJB equations are optimal, as indicated by Gershwin [34], Dehayem et al. [20], and references therein.

\section{Appendix B. Numerical approach}

Nevertheless, the HJB equations (9) generally lead to intractable solutions. Fortunately, Boukas and Haurie [32] have successfully found an approximate solution by applying the Kushner approach, reported in Kushner and Dupuis [35], where the central premise is to apply an approximation scheme for the gradient of the value function, $v(\alpha, x, a)$ by a discrete function $v^{h}(\alpha, x, a)$ and the partial derivatives $\frac{\partial v}{\partial x}$ and $\frac{\partial v}{\partial a}$ by:

$$
\frac{\partial v}{\partial x}(\alpha, x, a)= \begin{cases}\frac{1}{h_{x}}\left[v^{h}\left(\alpha, x+h_{x}, a\right)-v^{h}(\alpha, x, a)\right] & \text { if } \quad \dot{x} \geq 0 \\ \frac{1}{h_{x}}\left[v^{h}(\alpha, x, a)-v^{h}\left(\alpha, x-h_{x}, a\right)\right] & \text { if } \quad \dot{x}<0\end{cases}
$$

and

$$
\frac{\partial v}{\partial \mathrm{a}}(\alpha, x, a)=\frac{1}{h_{a}}\left[v^{h}\left(\left(\alpha, x, a+h_{a}\right)-v^{h}(\alpha, x, a)\right]\right.
$$

where $h_{x}$ and $h_{\mathrm{a}}$ indicate the length of the finite differential interval for the state variables $(x, a)$. Using the Kushner technique, the HJB equations (9) can be expressed in terms of the discrete function $v^{h}(\alpha, x, a)$ with step size $h_{x}$ and $h_{\mathrm{a}}$ on a discrete grid, as follows:

$$
\begin{aligned}
& v^{h}(\alpha, x, \mathrm{a})= \\
& \min _{\left(u_{1}, u_{2}, \omega_{o}\right) \in \Gamma(\alpha)}\left[( \rho + | \mathrm { q } _ { \alpha \alpha } | + \frac { | \mathrm { y } | } { h _ { x } } + \frac { k _ { 1 } \cdot u _ { 1 } } { h _ { a } } ) ^ { - 1 } \left(\varphi^{\alpha}(\cdot)+v^{h}\left(\alpha, \mathrm{x}, a+h_{a}\right) \frac{k_{1} \cdot u_{1}}{h_{a}}\right.\right. \\
& +v^{h}\left(\alpha, x+h_{x}, a\right) \frac{|y|}{h_{x}} \operatorname{Ind}\{\mathrm{y} \geq 0\}+v^{h}\left(\alpha, x-h_{x}, a\right) \frac{|y|}{h_{x}} \operatorname{Ind}\{\mathrm{y}<0\} \\
& \left.\left.+\sum_{\alpha \prime \neq \alpha} q_{\alpha \alpha^{\prime}}(\cdot) v\left(\alpha^{\prime}, x, \varphi(\xi, a)\right)\right)\right] \forall \alpha \in \Omega, \quad x \in R, a \in N
\end{aligned}
$$

where $y=u_{1}(t, \alpha) \cdot[1-\beta(\mathrm{a})]+u_{2}(t)-d$. Equation (B.3) is the discrete version of the HJB equations (9). The policy improvement method is used then to determine the structure of the control policy. The optimality of our results is a consequence of $\left(\mathrm{u}_{1}^{*}, \mathrm{u}_{2}^{*}, \omega_{\mathrm{o}}^{*}\right)$ and $v(\cdot)$ satisfying the HJB equation. The next theorem shows that $v^{h}(\alpha, x, a)$ is an approximation to $v(\alpha, x, a)$ for small step size $h$ (with $h=\left(h_{x}, h_{a}\right)$.

Theorem A.1. Let $v^{h}(\alpha, x, a)$ denotes a solution to HJB equations (9). Assume that there are constants $C_{g}$ and $\kappa_{g}$ such that 


$$
0 \leq v^{h}(\alpha, x, a) \leq C_{g}\left(1+|x|^{\kappa_{g}}\right)
$$

then

$$
\lim _{h \rightarrow 0} v^{h}(\alpha, x, a)=v(\alpha, x, a)
$$

Proof:

The proof of this theorem is similar to the one presented in Yan and Zhang [36].

\section{References}

[1] Akella, R. and Kumar, P.R., 1986, Optimal control of production rate in a failure prone manufacturing system, IEEE Transactions on Automatic Control, vol. AC31, pp. 116-126.

[2] Inman R.R., Blumenfeld D.E. and Huang, N., 2003, Designing production systems for quality: research opportunities from an automotive industry perspective, International Journal of Production Research, 41:9, pp. 1953-1971.

[3] Colledani, M., Tolio, T., Fisher, A., Iung, B., Lanza, G., Schmitt, R., Vancza, J., 2014, Design and management of manufacturing systems for production quality, CIRP Annals - Manufacturing Technology, vol. 63, issue 2, pp. 773-796.

[4] Kim, J. and Gershwin, S., 2005, Integrated quality and quantity modeling of a production line, OR Spectrum 27, pp. 287-314.

[5] Kim J. and Gershwin S., 2008, Analysis of long flow lines with quality and operational failures, IIE Transactions, 40, pp. 284-296.

[6] Colledani, M., Ebrahimi, D., Tolio, T., 2014, Integrated quality and production logistics modelling for the design of selective and adaptive assembly systems, CIRP Annals - Manufacturing Technology, vol. 63, issue 1, pp. 453-456.

[7] Radhoui, M., Rezg, N. and Chelbi, A., 2010, Joint quality control and preventive maintenance strategy for imperfect production processes, Journal of Intelligent Manufacturing, Vol. 21, issue 2, pp. 205-212.

[8] Mhada, F., Hajji, A., Malhame, R., Gharbi, A. and Pellerin, R., 2011, Production control of unreliable manufacturing systems producing defective items, Journal of Quality in Maintenance Engineering, Vol. 17, No. 3, pp. 238-253.

[9] Bouslah, B., Gharbi, A. and Pellerin, R., 2013, Joint optimal lot sizing and production control policy in an unreliable and imperfect manufacturing system, International Journal of Production Economics, vol. 144, no. 1 pp 143-156. 
[10] Dhouib, K., Gharbi, A., Ben-Aziza, M.N., 2012, Joint optimal production control/preventive maintenance policy for imperfect process manufacturing cell, International Journal of Production Economics, vol. 137, issue 1, pp. 126-136.

[11] Martinelli, F. and Piedimonte F., 2008, Optimal cycle production of a manufacturing system subject to deterioration, Automatica, 44, 2388-2391.

[12] Dehayem Nodem, F.I., Kenné, J.P. and Gharbi, A., 2011, Simultaneous control of production, repair/replacement and preventive maintenance of deteriorating manufacturing systems, International Journal of Production Economics, vol. 134, issue 1, pp. 271-282.

[13] Colledani, M. and Tolio, T., 2012, Integrated quality, production logistics and maintenance analysis of multi-stage asynchronous manufacturing systems with degrading machines, CIRP Annals-Manufacturing Technology, 61, pp. 455-458.

[14] Rivera-Gómez, H., Gharbi, A. and Kenné, J.P., 2013, Joint Production and major maintenance planning policy of a manufacturing system with deteriorating quality, International Journal of Production Economics, vol. 146, issue 2, 575587.

[15] Tan, B. and Gershwin, S.B., 2004, Production and Subcontracting strategies for manufacturers with limited capacity and volatile demand, Annals of Operations Research, 125, 205-232.

[16] Hajej, Z., Dellagi, S., Rezg, N., 2014, Joint optimization of maintenance and production policies with subcontracting and product returns, Journal of Intelligent Manufacturing, vol. 25, issue 3, pp. 589-602.

[17] Gharbi, A., Hajji, A., and Dhouib, K., 2011, Production rate control of an unreliable manufacturing cell with adjustable capacity, International Journal of Production Research, 49:21, 6539-6557.

[18] Dror, M., Kenneth, R.S., Candace A.Y., 2009, Deux Chemicals Inc. Goes Just-inTime, Interfaces, vol. 39, issue 6, pp. 503-515.

[19] Assid, M., Gharbi, A., Dhouib. K., 2015, Joint production and subcontracting planning of unreliable multi-facility multi-product production systems, Omega, vol 50, pp. 54-69.

[20] Dehayem Nodem, F.I., Kenne, J.P., Gharbi, A., 2011, Simultaneous control of production, repair/replacement and preventive maintenance of deteriorating manufacturing systems, International Journal of Production Economics, vol. 134, issue 1, pp. 271-283.

[21] Kouedeu A.F., Kenne J.P., Dejax, P., Songmene, V., Polotski, V., 2014, Stochastic optimal control of manufacturing systems under production-dependent 
failure rates, International Journal of Production Economics, vol. 150, pp. 174187.

[22] Rosenblatt, M. J., and Lee, H. L. 1986, Economic production cycles with imperfect production processes. IIE transactions, vol. 18, issue 1, pp. 48-55.

[23] Sana, S. S. 2010. An economic production lot size model in an imperfect production system. European Journal of Operational Research, vol. 201, issue 1, pp. $158-170$.

[24] Colledani, M. and Tolio, T., 2011, Integrated analysis of quality and production logistics performance in manufacturing lines, International Journal of Production Research vol. 49, issue 2, pp. 485-518.

[25] Love, C.E., Zhang, Z.G., Zitron, M.A., Guo, R., 2000, A discrete semi-Markov decision model to determine the optimal repair/replacement policy under general repairs, European Journal of Operational Research, 125 pp. 398-409.

[26] Kenne, J.P. and Gharbi, A., 1999, Experimental design in production and maintenance control problem of a single machine, single product manufacturing system, International Journal of Production Research, vol. 37, issue 3, pp. 621637.

[27] Njike, A.N., Pellerin, R., Kenne, J.P., 2011, Maintenance/production planning with interactive feedback of product quality, Journal of Quality in Maintenance Engineering, vol. 17, no. 3, pp. 281-298.

[28] BenSalem, A., Gharbi, A. and Hajji, A., 2015, Environmental issue in an alternative production-maintenance control for unreliable manufacturing system subject to degradation, International Journal of Advanced Manufacturing Technology, vol. 77, issue 1-4, pp. 383-398.

[29] Bielecki, T. and Kumar, P.R., 1988. Optimality of zero-inventory policies for unreliable manufacturing systems. Operations Research, Vol. 36, No.4, pp. 532541.

[30] Olsder, G.J., and Suri, R., 1980. Time-optimal control of parts-routing in a manufacturing system with failure-prone machines. $19^{\text {th }}$ IEEE Conference on Decision and Control including the Symposium on Adaptive Processes, pp. 722727.

[31] Wang, Z. and Gershwin, S.B., 2015. Heuristic production and sale policy for a two-product-type manufacturing system with downward substitution. IEEE Transactions on systems, man. and cybernetics, vol. 45, no.6, pp. 929-942.

[32] Boukas, E.K. and Hauire, A., 1990, Manufacturing flow control and preventive maintenance: a stochastic control approach, IEEE Transactions on Automatic Control 33, pp. 1024-1031. 
[33] Gershwin, S. B., 2002. Manufacturing Systems Engineering. Cambridge, MA: Massachusetts Institute of Technology, p. 350. Second private printing.

[34] Gershwin, S. B., 2000, Design and operation of manufacturing systems: the control-point policy, IIE Transactions, 32, pp. 891-906.

[35] Kushner, H.J. and Dupuis, P.G., 1992, Numerical Methods for Stochastic Control Problems in Continuous Time, (Springer, New York, NY).

[36] Yan H. and Zhang Q. (1997). A numerical method in optimal production and setup scheduling to stochastic manufacturing systems, IEEE Transactions on Automatic Control, 42, 1452-1455. 